University of Wollongong

Research Online

Faculty of Engineering and Information

Faculty of Engineering and Information

Sciences - Papers: Part A

Sciences

$1-1-2014$

\title{
Distribution expansion planning considering reliability and security of energy using modified PSO (Particle Swarm Optimization) algorithm
}

Jamshid Aghaei

Shiraz University of Technology, jamshid@uow.edu.au

Kashem M. Muttaqi

University of Wollongong, kashem@uow.edu.au

Ali Azizivahed

Shiraz University of Technology

Mohsen Gitizadeh

Shiraz University of Technology

Follow this and additional works at: https://ro.uow.edu.au/eispapers

Part of the Engineering Commons, and the Science and Technology Studies Commons

Research Online is the open access institutional repository for the University of Wollongong. For further information contact the UOW Library: research-pubs@uow.edu.au 


\title{
Distribution expansion planning considering reliability and security of energy using modified PSO (Particle Swarm Optimization) algorithm
}

\begin{abstract}
Distribution feeders and substations need to provide additional capacity to serve the growing electrical demand of customers without compromising the reliability of the electrical networks. Also, more control devices, such as DG (Distributed Generation) units are being integrated into distribution feeders.

Distribution networks were not planned to host these intermittent generation units before construction of the systems. Therefore, additional distribution facilities are needed to be planned and prepared for the future growth of the electrical demand as well as the increase of network hosting capacity by DG units. This paper presents a multiobjective optimization algorithm for the MDEP (Multi-Stage Distribution Expansion Planning) in the presence of DGs using nonlinear formulations. The objective functions of the MDEP consist of minimization of costs, END (Energy-Not-Distributed), active power losses and voltage stability index based on SCC (Short Circuit Capacity). A MPSO (modified Particle Swarm Optimization) algorithm is developed and used for this multiobjective MDEP optimization. In the proposed MPSO algorithm, a new mutation method is implemented to improve the global searching ability and restrain the premature convergence to local minima. The effectiveness of the proposed method is tested on a typical 33-bus test system and results are presented.
\end{abstract}

\section{Keywords}

considering, planning, reliability, expansion, security, particle, distribution, pso, energy, modified, algorithm, optimization, swarm

\section{Disciplines}

Engineering | Science and Technology Studies

\section{Publication Details}

J. Aghaei, K. M. Muttaqi, A. Azizivahed \& M. Gitizadeh, "Distribution expansion planning considering reliability and security of energy using modified PSO (Particle Swarm Optimization) algorithm," Energy, vol. 65, pp. 398-411, 2014. 


\title{
Distribution Expansion Planning considering Reliability and Security of Energy
}

\section{Using Modified PSO Algorithm}

\author{
Jamshid Aghaei ${ }^{1}$, Kashem M. Muttaqi ${ }^{2}$, Ali Azizivahed ${ }^{1}$, and Mohsen Gitizadeh $^{1}$ \\ ${ }^{1}$ Department of Electronics and Electrical Engineering, Shiraz University of Technology, Shiraz, Iran \\ ${ }^{2}$ Integral Energy Power Quality and Reliability \\ Centre, School of Electrical, Computer and Telecommunications Engineering, University of Wollongong
}

Corresponding Author: J. Aghaei (aghaei@sutech.ac.ir); Mail Address: Room No. 199, Department of Electrical and Electronics Engineering, Shiraz University of Technology, Modarres Blvd., Shiraz, Iran, Postal Code: 71557-13957.

\footnotetext{
Abstract-Distribution feeders and substations need to provide additional capacity to serve the growing electrical demand of customers without compromising the reliability of the electrical networks. Also, more control devices, such as Distributed Generation (DG) units are being integrated into distribution feeders. Distribution networks were not planned to host these intermittent generation units before construction of the systems. Therefore, additional distribution facilities are needed to be planned and prepared for the future growth of the electrical demand as well as the increase of network hosting capacity by DG units. This paper presents a multiobjective optimization algorithm for the Multi-Stage Distribution Expansion Planning (MDEP) in the presence of DGs using nonlinear formulations. The objective functions of the MDEP consist of minimization of costs, Energy-Not-Distributed (END), active power losses and voltage stability index based on Short Circuit Capacity (SCC). A modified PSO (MPSO) algorithm is developed and used for this multiobjective MDEP optimization. In the proposed MPSO algorithm, a new mutation method is implemented to improve the global searching ability and restrain the
} 
premature convergence to local minima. The effectiveness of the proposed method is tested on a typical 33-bus test system and results are presented.

Index Terms- Multi-stage Distribution Expansion Planning (MDEP); Energy-Not-Distributed (END); Short Circuit Capacity (SCC); Modified PSO (MPSO); Multiobjective Optimization.

\section{I.Introduction}

Expansion planning of distribution networks seeks the best reinforcement plans while minimizing the overall cost subject to various operational and reliability constraints. Also, it is one of the complex problems with several decades' history of continuous efforts and contributions for improved solutions. Distribution Expansion Planning (DEP) is an important issue in developing countries all over the world, whose electricity demand has been boosting up in recent years. However, significant efforts in energy management realm have recently damped the increasing rate of electricity demand. Nonetheless, the need for a continuous expansion seems inevitable in the near future. Expansion planning of distribution network consists of determining the capacity, installation and/or reinforcement of distribution substation units, installing Distributed Generation (DG) units, and addition or replacement of distribution feeders to serve future increasing load demand. This, so called Multi-Stage Distribution Expansion Planning (MDEP) problem, can be formulated as a nonlinear problem with nonlinear constraints involving many local optimum solutions.

Furthermore, in the distribution network expansion, a reliable energy supply with predefined satisfaction level should be provided for customers. Thus, network reliability is one of the major issues to be considered in MDEP formulation. Accordingly, the index of Energy Not Distributed (END) is calculated to count on the network reliability which is very complex and relates to many factors such as geographical structure of the distribution network, number of faults in each branch, and reparation and restoration time of the branches.

Today, the distribution networks are equipped with new control devices such as DGs. Using local DGs by utility customers leads to avoid unnecessary expansion of distribution networks as well as more efficient 
use of the existing networks. Customers in the portions of the distribution grid where capacity constraints are imminent could in the future be provided with incentives to generate some of their own power (or shed some of their loads), especially at the period of peak power demand. Also, DG offers the additional advantage of increase in reliability levels. Furthermore, using DGs can affect the network power losses due to its proximity to the load centers. Therefore, optimal sizing and siting of DG units should be included as a part of the MDEP problem.

Distribution networks are characterized by a designed SCC and a maximum acceptable fault current, which are related to the used switchgear and the thermal and mechanical withstand capability of the equipment and constructions as well. High integration of DGs to the system should not lead to exceeding the designed fault current level of the network [1]. Accordingly, considering short circuit capability of the system is of importance for the MDEP problem in the presence of DG integration.

Due to the demand growth, generation intermittency and network capacity limitation, research interest on Distribution Expansion Planning (DEP) has been growing. In [2-5], DEP problem is solved for only one planning horizon. In these papers, the location, type, and the capacity of new equipment are evaluated. In [6-7], DEP problem is solved for several stages. In these works, the growth of the demand for a long time is evaluated. The MDEP has been solved using Mixed Integer Linear Programming (MILP) framework in [8-12]. In [7, 13-16], the evolutionary algorithms are used for the solution of the DEP problem. In [17], the graph theory is used to formulate MDEP. Solving MDEP problem using Optimal Power Flow (OPF) and Genetic Algorithm (GA) is proposed in [18]. Distribution system planning for peak cutting using GA is used for minimizing investment cost in [19]. In [20], a multiobjective decision making procedure is applied to MDEP problem using an efficient heuristic search method to minimize the total cost while the associated technical constraints are satisfied. Reliability indices of a radial distribution network have been improved using multiple fault indicators and optimum DG placement in [21] and [22], respectively. Reliability-oriented distribution network reconfiguration considering demand uncertainty has been studied in [23]. The short-circuit contribution of the various DG types is extensively discussed in [24]. Fundamental issues related to the interconnection of DG installations to the grid as well as evaluation 
rules are discussed in [25]. In [26], a method is proposed to determine the optimum allocation of the maximum DG penetration in the medium voltage power distribution networks.

There are some research works in the area which deal with Distribution Feeder Reconfiguration (DFR) problem [27-32]. For instance, multi-objective adaptive PSO algorithms have been presented in [27, 28] to solve stochastic DFR problem for systems with distributed wind power generation and fuel cells. Also, in $[29,30]$, the effect of DFR on the operating management of fuel cell power plants has been assessed in a stochastic environment using point estimate method. Besides, a new hybrid fuzzy adaptive PSO and Nelder-Mead simplex search method [31], and honey bee mating optimization algorithm [32] have been presented to solve the DFR problem in [31, 32]. It is noted that the operation management of distribution networks has been assessed in [27-32]. However, in this paper, the planning issue has been concentrated for distribution networks. Moreover, reliability and voltage stability indicators have been included in the proposed framework in this paper.

This paper formulates a new multiobjective, multi-stage expansion planning of distribution networks using Modified Particle Swarm Optimization (MPSO) algorithm. DG units are included in MDEP problem as discussed previously. Investment and Operation (I\&O) costs, energy not distributed, electrical power losses, and voltage stability based on SCC are used to develop the objective functions in this paper. Furthermore, a new approach based on graph theory for checking radial structure of distribution network is proposed. Accordingly, to the best of our knowledge, the contributions of this paper with respect to previous ones in the area can be summarized as follows: (i) including reliability index, i.e., END and voltage stability index based on SCC as new extra objective functions in the MDEP formulation; (ii) using MPSO as optimization solution algorithm; (iii) using graph theory to assess the radial structure of the network; (iv) including AC power flow constraints in MDEP problem; and (v) implementing some indices to evaluate the Pareto solutions.

The remainder of this paper is organized as follows: Section II presents the mathematical formulation of the objective functions of the MDEP problem. In the next section, the application of MPSO for multiobjective MDEP is presented. Section IV is devoted to present the numerical results. A distribution 
system with 33 buses is used as a test system to verify the applicability and validity of the proposed approach. Finally, some relevant conclusions are drawn in Section V.

\section{Problem formulation}

Main target of conventional MDEP is to expand the structure of distribution network to provide increasing electrical demand. Therefore, MDEP refers to the reinforcement or installations of new distribution substations, replacement of old feeders and construction of new feeders for feeding new buses, and also optimal placement of DG units. Accordingly, the multiobjective MDEP problem can be formulated as follows:

\section{A. Objective functions}

The multiobjective MDEP problem consists of four competing objectives, which are I\&O cost, END, power losses, and voltage stability based on SCC.

\section{Minimization of $I \& O$ cost}

Investment cost and operation cost are formulated and defined as per the expressions given in (1) and (2), respectively.

$$
\begin{aligned}
& \operatorname{Cost}_{\text {Investment }}(X)=\sum_{t=1}^{N_{\text {Stuge }}} c_{N P V}^{t}\left(\sum_{k=1}^{N_{B}^{t}} I C_{k}+\sum_{s=1}^{N_{S}^{t}} I C_{s}+\sum_{d g=1}^{N_{D}^{t}} I C_{d g}\right) \\
& \operatorname{Cost}_{\text {Operation }}(X)=\sum_{t=1}^{N_{\text {Stage }}} c_{N P V}^{t}\left(\sum_{k=1}^{N_{B}^{t}} O C_{k}+\sum_{s=1}^{N_{S}^{t}} O C_{s}+\sum_{d g=1}^{N_{D}^{t}} O C_{d g}\right)
\end{aligned}
$$

Where, $N_{B}^{t}, N_{S}^{t}, N_{D}^{t}$ and $N_{\text {Stage }}$ are the number of all branches (exiting and added branches), number of substations, number of distribution generation units and number of stages in the planning horizon, respectively.

Where, $X$ denotes the set of decision variables that should be specified by optimization algorithm. 
The objective function for cost optimization can be written as,

$F_{1}(X)=$ Cost $_{\text {Investment }}+$ Cost $_{\text {Operation }}$

Set of decision variables of MDEP optimization problem can be summarized as follows:

$X=\left[\begin{array}{llll}x_{C} & X_{R} & x_{A} & x_{D}\end{array}\right]$

$x_{C}=\left[\begin{array}{llll}x_{b 1} & x_{b 2} & \ldots & x_{b N_{E B}}\end{array}\right]$

$x_{R}=\left[\begin{array}{llll}x_{r 1} & x_{r 2} & \ldots & x_{r N_{R B}}\end{array}\right]$

$x_{A}=\left[\begin{array}{llll}x_{a 1} & x_{a 2} & \ldots & x_{a N_{A B}}\end{array}\right]$

$x_{D}=\left[\begin{array}{llll}x_{d 1} & x_{d 2} & \cdots & x_{d N_{D}}\end{array}\right]$

$N_{B}=N_{E B}+N_{A B}$

$\left\{x_{R}\right\} \in\left\{x_{C}\right\}$

Where, $x_{C}, x_{R}$ and $x_{A}$, respectively, are a set of existing network, a set of branches which needed rewiring and a set of branches which installed for feeding the new busses. $x_{D}$ is set of decision variables for sizing and placement of DG's. Also, $N_{E B}, N_{R B}$ and $N_{A B}$ are the number of exiting branches, number of replacing branches and number of added branches, respectively.

\section{Minimization of END}

Most power outages are caused by faults in the transmission and distribution networks. Accordingly, the MDEP problem should be carried out to optimize a reliability index such as minimization of END as follows:

$$
\begin{aligned}
& E N D_{i}=P_{i} \sum_{i, j \in V, j \neq i}\left(U_{j, i}+U_{j, i}^{\prime}\right) \\
& E N D(X)=\sum_{t=1}^{N_{\text {Suge }}} \sum_{j=1}^{N_{\text {bus }}^{t}} E N D_{j}^{t} \\
& F_{2}(X)=E N D
\end{aligned}
$$


Where, $U_{j, i}$ and $U_{j, i}^{\prime}$ are the service unavailability related to the reparation time of all the upward branches connected to the bus $i$ and the service unavailability associated to the restoration time of all the downward branches connected to the bus $i$, respectively [33-34]. They can be formulated in the following mathematical model:

$U_{j, i}=\beta_{j, i} \times t_{j, i}$

$U_{j, i}^{\prime}=\beta_{j, i} \times t_{j, i}^{\prime}$

Where, $\beta_{j, i}$ is failure rate (fail/year) of a branch connecting the nodes $\mathrm{j}$ to I, i.e., branch $_{\mathrm{j}, \mathrm{i}} t_{j, i}$ and $t_{j, i}^{\prime}$ are average reparation and restoration time (h), respectively.

To have a better illustration of the END, a simple distribution system, as shown in Fig. 1, is used as an example. For instance, the END of bus $6\left(\mathrm{END}_{6}\right)$ can be determined as follows: if there is a failure at the branch $_{1,5}$ and branch ${ }_{5,6}$, the electrical power to feed bus ${ }_{6}$ will be interrupted, since these branches should be repaired, and if there is failure at the branch $_{6,7}$ and branch $_{6,8}$, the electrical power to feed bus 6 will be stopped while the sectionalizing switch of the feeder would be closed. Therefore, the $\mathrm{END}_{6}$ can be formulated as:

$E N D_{6}=P_{6} \times\left(U_{1,5}+U_{5,6}+U_{6,7}^{\prime}+U_{6,8}^{\prime}\right)$

\section{Minimization of power losses}

The third objective is to minimize the total active power losses for expansion planning horizon, which can be modeled as:

$$
\begin{aligned}
& P_{\text {Loss }}(X)=\sum_{t=1}^{N_{\text {Slage }}} \sum_{k=1}^{N_{\text {trct }}^{t}}\left(R_{k}^{t} \times\left|I_{k}^{t}\right|^{2}\right) \\
& F_{3}=P_{\text {Loss }}
\end{aligned}
$$

Where, $R_{k}^{t}$ and $I_{k}^{t}$ are the resistance and the magnitude of current for the $k^{\text {th }}$ branch in the $t^{\text {th }}$ stage, respectively.

\section{Minimizing voltage stability index (VSI) based on SCC}


Improving the SCC of distribution network in the presence of DG units is one of important goals of the distribution system planners for distribution expansion problem. The SCC directly relates to the substation bus voltage strength. Indeed, a high SCC means the bus is able to connect more loads [35-36], and on the other hand a low SCC means the network is weak [37].

Based on the Thevenin equivalent system of bus $j$, as illustrated in Fig.2, the SCC, i.e., $S_{s c}$, of bus $j$ is calculated as follow:

$S_{s c, j}=E_{t h, j} / Z_{t h, j}$

Where $E_{t h, j}$ and $Z_{t h, j}$ are Thevenin equivalent voltage and impedance of bus $j$, respectively. Minimal SCC to ensure voltage stability of bus $j$ is deduced in [38] and it can be obtained as follows:

$$
\begin{aligned}
& S_{s c \min , j}=\frac{2 S_{L j}\left(1+\sin \theta_{j}\right)}{E_{t h j}} \\
& S_{L j}=\sqrt{{P_{L j}{ }^{2}+Q_{L j}{ }^{2}}^{2}}
\end{aligned}
$$

Where, $\theta_{j}$ is power factor angle. Also, $P_{L j}$, $Q_{L j}$ and $S_{L j}$ are the active, reactive and apparent power of bus $j$, respectively.

The relationship between voltage stability and SCC can be described as below:

If $\frac{S_{s c \min , j}-S_{s c, j}}{S_{s c, j}}<0$, then the voltage of bus $j$ is stable.

If $\frac{S_{s c \min , j}-S_{s c, j}}{S_{s c, j}}>0$, then the voltage of bus $j$ is unstable.

The voltage stability index, $I_{S C C}$, of bus $j$ based on SCC is defined as [37]:

$$
\begin{aligned}
& I_{S C C, j}=\frac{S_{s c \min , j}}{S_{s c}} \\
& F_{4}=\sum_{t=1}^{N_{\text {Stage }}} \frac{1}{N_{\text {bus }}^{t}} \sum_{i=1}^{N_{\text {bus }}^{t}} I_{S C C, i}^{t}
\end{aligned}
$$

It is obvious that $F_{4}$ should be minimized. 


\section{B. Constraints}

The MDEP problem is subject to the following constraints:

- Voltage limits of buses

$$
v_{j}^{\min } \leq v_{j} \leq v_{j}^{\max }
$$

Where, $v_{j}, v_{j}^{\min }$ and $v_{j}^{\max }$, are the magnitude, minimum and maximum value of voltage at bus $j$.

- Power flow transmission of feeders and branches

$$
P F_{k} \leq P F_{k}^{\max }
$$

where, $P F_{k}$ and $P F_{k}^{\max }$, are the power flow amount of branch $k$ and its maximum allowable power flow.

- Power output of DG

$$
P_{d g} \leq P_{d g}^{\max }
$$

where, $P_{d g}$ and $P_{d g}^{\max }$, are the power output of $d g^{\text {th }}$ distribution generation and its maximum capacity.

- Radial structure of the distribution network

The structure of distribution system should be radial due to the simpler protection schemes of distribution networks. For this purpose, branch-bus incidence matrix is used for checking radial structure of networks. The branch-bus incidence matrix of $A$ is a $N_{\text {branch }} \times N_{\text {bus }}$ matrix wherein the $k^{\text {th }}$ row of the matrix

corresponds to the $k^{\text {th }}$ branch in the network and the $j^{\text {th }}$ column of the matrix corresponds to the $j^{\text {th }}$ bus in the system which has a branch leaving the bus. Incidence matrices are mostly used in graph theory [39]. The branch-bus incidence matrix is calculated as follows:

- If the $k^{\text {th }}$ branch (corresponding to $k^{\text {th }}$ row) leaves from $j^{\text {th }}$ bus (corresponding to $j^{\text {th }}$ column) then the matrix element $\left(a_{k j}\right)$ is equal to 1 .

- If the $k^{\text {th }}$ branch (corresponding to $k^{\text {th }}$ row) inters toward $j^{\text {th }}$ bus (corresponding to $j^{\text {th }}$ column) then the matrix element $\left(a_{k j}\right)$ will be -1 .

- $\quad$ All remaining entries will be identically zero. 
- While the number of buses is one more than the number of branches in the radial distribution networks, the first column of branch-bus incidence matrix $A$ should be deleted to have a square matrix $A^{\prime}$.

- If the determinant of branch-bus incidence matrix $A^{\prime}$ is 1 or -1 , the network's graph will be radial.

\section{Multiobjective solution methodology}

In the following, the implemented solution methodology based on the MPSO algorithm has been presented. Subsequently, the multiobjective solution strategy has been addressed.

\section{A. Modified PSO}

In the original PSO algorithm, each individual element is called a "particle", and is subject to a movement in a multi-dimensional space. This algorithm inspired based on the simulation of the food searching activities of a flock of birds [40]. Particles have memory, thus they retains their previous state. Each particle's movement is the composition of an initial random velocity and two randomly weighted influences: individuality, the tendency to return to the particle's best previous position, and sociality, and the tendency to move towards the neighborhood's best previous position.

It is noted that the PSO [40] is known as an optimization algorithm which has the ability to escape from local optima by accepting non-improving energy solution during the first and middle stage of the algorithm. Also, the PSO is widely-used algorithm in the literature of power systems due to its simple implementation. Besides, in this paper, to improve the ability of PSO algorithm to avoid early local convergence, different mutation strategies have been proposed. Indeed, by implementing mutation operator, individual elements and particles are randomly altered. Therefore, the modified PSO is proper algorithm to solve the DEP problem which is a complicated non-linear and non-convex problem with many local optima.

The mathematical model for the PSO is as follows:

$v_{i}^{i t e r+1}=k \times\left[\omega \times v_{i}^{i t e r}+c_{1} \times \operatorname{rand}() \times.\left(x_{i}^{\text {best }}-x_{i}^{\text {iter }}\right)+c_{2} \times \operatorname{rand}() \times.\left(x^{\text {Gbest }}-x_{i}^{\text {iter }}\right)\right]$ 
$x_{i}^{i t e r+1}=x_{i}^{i t e r}+v_{i}^{\text {iter }+1}$

Where, $x_{i}^{\text {best }}$ and $x^{\text {Gbest }}$ are the best personal experience and global experience of particles, respectively. $x_{i}^{j}$ is the $i^{\text {th }}$ particle position in the $i t e r^{\text {th }}$ iteration, and $v_{i}^{\text {iter }}$ is the $i^{\text {th }}$ particles velocity in the $i t e r^{\text {th }}$ iteration. $\omega$ is inertia weighting, $c_{1}$ and $c_{2}$ are and accelerating parameters. Also, rand(.) is random number generator between 0 and 1 . The term $\mathrm{k}$ is defined as:

$k=\frac{2}{\left|2-C-\sqrt{C^{2}-4 \times C}\right|}$

Where, $C=c_{1}+c_{2}$ and $C>4$.

In this paper, to improve the ability of PSO algorithm to avoid early local convergence, different mutation strategies have been proposed. Indeed, by implementing mutation operator, individual elements and particles are randomly altered. The purpose of the mutation in PSO algorithm is preserving and introducing diversity. Mutation should allow the algorithm to avoid local optima by preventing the population of particles from becoming too similar to each other. Without employing mutation, the evaluation of particles may be slowed or even stopped. The mutant particle is generated as follows [4142]:

$$
\begin{aligned}
& X_{\text {mut }}^{1}=x^{\text {Gbest }}+\psi \times\left(X_{\text {rand } 1}-X_{\text {rand } 2}\right)+\psi \times\left(X_{\text {rand } 3}-X_{\text {rand } 4}\right) \\
& X_{\text {mut }}^{2}=X^{\text {Gbest }}+\psi \times\left(x_{\text {rand 1 }}-x_{\text {rand } 2}\right) \\
& X_{\text {mut }}^{3}=x_{\text {rand } 3}^{\text {iter }}+\psi \times\left(x^{\text {Gbest }}-x_{\text {rand } 3}^{\text {iter }}\right)+\psi \times\left(x_{\text {rand } 1}-x_{\text {rand } 2}\right) \\
& x_{\text {mut }}^{4}=x_{\text {rand } 1}+\operatorname{rand}(.) \times\left(x_{4}^{\text {best }}-x_{\text {rand } 2}\right)+\operatorname{rand}(.) \times\left(x^{\text {Gbest }}-x_{\text {rand } 2}\right) \\
& X_{\text {mut }}^{5}=X_{\text {rand } 1}+\psi \times\left(X_{\text {rand } 2}-X_{\text {rand } 3}\right)+\psi \times\left(X_{\text {rand } 4}-X_{\text {rand } 5}\right)
\end{aligned}
$$

Where, $x_{\text {rand } 1} \neq X_{\text {rand } 2} \neq x_{\text {rand } 3} \neq X_{\text {rand } 4} \neq x_{\text {rand } 5}$ are randomly selected mutant particles. $\psi$ is the mutation constant that its value usually is equal to 2 [41]. To employ five mutant particles, 5 
particles $\left(x_{\text {sele }}^{1}, X_{\text {sele }}^{2}, X_{\text {sele }}^{3}, X_{\text {sele }}^{4}, X_{\text {sele }}^{5}\right)$ will be randomly selected in each iteration. If the generation cost of the $F\left(x_{\text {mut }}^{r}\right), r=1,2, \ldots, 5$ is better than that of the $F\left(x_{\text {sele }}^{r}\right)$, the selected particle $x_{\text {sele }}^{r}$ is replaced by the mutant particle $x_{\text {mut }}^{r}$ in the next iteration. Otherwise, $x_{\text {sele }}^{r}$ will be remained in the next iteration.

\section{B. Multiobjective strategy}

Multiobjective optimization is the process of simultaneously optimizing two or more conflicting objective functions subject to certain constraints. In these cases, the decision makers are looking for the "most preferred” solution. In MMP, the concept of optimality is replaced with that of efficiency or Pareto optimality. The efficient (or Pareto optimal, non-dominated, non-inferior) solution is the solution that cannot be improved in one objective function without deteriorating its performance in at least one of the rest. In other words, the solution $X_{2}$ is dominated by $X_{1}$, when the following conditions are met [43]:

$$
\begin{aligned}
& \forall m \in\left\{1, \quad 2, \quad \ldots, \quad, N_{O b j}\right\}, F_{m}\left(X_{1}\right) \leq F_{m}\left(X_{2}\right), \\
& \exists n \in\left\{1, \quad 2, \quad \ldots \quad, N_{O b j}\right\}, F_{n}\left(X_{1}\right)<F_{n}\left(X_{2}\right),
\end{aligned}
$$

In the multiobjective optimization, usually the scale of objective functions is not the same. For this purpose, a fuzzy approach is used to normalize the objective functions. In this approach, each objective function is modeled by descending and smooth membership function as follows:

$$
\mu_{F m}(X)=\left\{\begin{array}{cll}
1 & , & F_{m}(X) \leq F_{m}^{\min } \\
0 \quad & , & F_{m}(X) \geq F_{m}^{\max } \\
\frac{F_{m}^{\max }-F_{m}(X)}{F_{m}^{\max }-F_{m}^{\min }}, & F_{m}^{\min } \leq F_{m}(X) \leq F_{m}^{\max }
\end{array} \quad m=1,2, \ldots, N_{O b j}\right.
$$

In the multiobjective PSO algorithm, the non-dominated solutions are saved in the repository in all iterations. For each individual in the repository, an evaluation criterion is calculated as follows: 
$N_{\mu}(i)=\frac{\sum_{m=1}^{N_{\text {obj }}} w_{m} \times \mu_{F_{m}}\left(X_{i}\right)}{\sum_{i=1}^{N_{\text {rep }}} \sum_{m=1}^{N_{\text {obj }}} w_{m} \times \mu_{F_{m}}\left(X_{i}\right)}$

Where, $N_{\text {rep }}$ is the number of individuals in the repository. Also, $w_{\mathrm{m}}$ refers to the impotence (or preference or priority) degree of the $m^{\text {th }}$ objective function from the viewpoint of Decision Maker (DM). In this approach, the membership functions are normalized. Also, in the repository, non-dominated solutions are sorted on the basis of $N_{\mu}$ using (34) and the importance of objective functions defined by the DM.

\section{Solution algorithm}

This section presents the application of the proposed algorithm to solve the proposed multiobjective MDEP problem. The steps of the algorithm are as follows:

Step 1: Define the input data. (Including network data and adjusting parameters of algorithm)

Step 2: Generate the initial population; Initial population is randomly generated considering the defined information in the previous step as follows:

$$
\begin{aligned}
& \text { Population }=\left[\begin{array}{c}
X_{1} \\
X_{2} \\
\ldots \\
X_{N p o p}
\end{array}\right] \\
& X=\left[\begin{array}{llll}
x_{C} & x_{R} & x_{A} & x_{D}
\end{array}\right] \\
& x_{C}=\left[\begin{array}{llll}
x_{b 1} & x_{b 2} & \ldots & x_{b N_{E B}}
\end{array}\right] \\
& x_{R}=\left[\begin{array}{llll}
x_{r 1} & x_{r 2} & \ldots & x_{r N_{R B}}
\end{array}\right] \\
& x_{A}=\left[\begin{array}{llll}
x_{a 1} & x_{a 2} & \ldots & x_{a N_{A B}}
\end{array}\right] \\
& x_{D}=\left[\begin{array}{llll}
x_{d 1} & x_{d 2} & \ldots & x_{d N_{D}}
\end{array}\right]
\end{aligned}
$$


Step 3: calculate the branch-bus incidence matrix $\left(A^{\prime}\right)$ and check the radial structure, if the structure of network is radial, go to the next step, otherwise add penalty term. In this paper, in the case of ring structure of networks, the objective functions are substituted by the penalty term which is a big number.

Step 4: Employ the load flow calculations using the well-known method proposed by [44]; based on the decision variables in each particle, a distribution load flow is solved. Then the problem constraints should be checked considering the results of the load flow. If the problem constraints are satisfied, go to the next step, otherwise add penalty term (a big number) as done in step 3.

Step 5: Compute and normalize the objective functions. The objective functions are calculated by Eqs. (1) to (20), using the results of the load flow. Then the fuzzy approach, i.e., Eq. (33), is used to obtain $\mu_{1}, \mu_{2}$ and $\mu_{3}$ corresponding to $F_{1}, F_{2}$ and $F_{3}$ respectively.

Step 6: Eq. (34) is used to compute the fitness function of each individual particle. Steps 3 to 6 are repeated for all members of initial population.

Step 7: Sort the particles in descending order of fitness value and determine the $X_{i}^{\text {best }}$ and $X^{\text {Gbest }}$, then save the non-dominated solutions in the repository.

Step 8: Update the particles using Eq. (24) and Eq. (25).

Step 9: Calculate the branch-bus incidence matrix $\left(A^{\prime}\right)$ and check the radial structure for all particles, if the structure of network is radial, go to the next step, otherwise add penalty term.

Step 10: Calculate the load flow; based on the decision variables in each particle, a distribution load flow is solved. Check the problem constraints considering the results of load flow for all particles, if the problem constraints are satisfied, go to the next step; otherwise add penalty term.

Step 11: Compute and normalize the objective functions, and then compute the fitness function.

Step 12: Apply the mutation process, which is described in section III. A.

Step 13: Sort the particles in descending order of fitness value and determine the $X_{i}^{\text {best }}$ and $X^{\text {Gbest }}$, then save the non-dominated solution in repository. Steps 8 to 13 are repeated until predetermined iteration number is reached. 
The flowchart of the proposed algorithm for solving the MDEP is shown in Fig. 3.

\section{Evaluating the performance of multiobjective optimization}

There are a variety of methods to characterize the Pareto performance. Three goals of Pareto multiobjective optimization that can be identified and measured are as follows:

- Distance of the resulting non-dominated set to the true Pareto front should be minimized.

- A good distribution of the obtained solutions.

- The size of the obtained non-dominated front should be maximized.

These performance metrics are described as follows:

Spacing metric: This metric, SP, has been introduced by Schott in [45]. The purpose of this metric is to gauge how evenly the points in the approximation set are distributed in the objective space. This metric is given by:

$$
\begin{aligned}
& S P=\sqrt{\frac{1}{N_{\text {rep }}-1} \sum_{i=1}^{N_{\text {rep }}}\left(\bar{d}-d_{i}\right)^{2}} \\
& d_{i}=\min _{j=1,2, \ldots, N_{\text {rep }}} i \neq j \\
& \bar{d}=\frac{1}{N_{\text {rep }}} \sum_{i=1}^{N_{\text {rep }}} d_{i}
\end{aligned}
$$

This metric displays a distance between the resulting non-dominated set and the real Pareto front. Therefore, the lower values of this parameter are more desirable.

Generational distance: This metric, GD, finds the average distance of solutions from the Pareto front [46]. This metric is explained as follows:

$G D=\frac{\sqrt{\sum_{i=1}^{N_{\text {rep }}} d_{i}^{2}}}{N_{\text {rep }}}$

where, parameter $d_{i}$ is the Euclidean distance (in the objective space) between each of these nondominated solution vectors and the nearest member of the Pareto-optimal set as defined in (42). This metric displays an average distance of the non-dominated solution in Pareto set. If this metric be close to zero, it shows that the obtained Pareto result is close to the Pareto-optimal set. 
Diversity metric: It is another metric, D, for the obtained Pareto solutions. The higher value of this metric shows the higher diversity of the obtained Pareto-optimal solutions.

$$
\begin{aligned}
D & =\sum_{m=1}^{N_{\text {obj }}} \sum_{i=1}^{N_{\text {rep }}}\left(F_{m}^{i}-C_{i}\right)^{2} \\
C_{i} & =\frac{\sum_{i=1}^{N_{\text {rep }}} F_{m}^{i}}{N_{\text {rep }}}
\end{aligned}
$$

\section{Results and Discussions}

The proposed multiobjective MDEP problem considering DGs was applied to a 33-bus distribution network test system. This system is the "Baran and Wu" distribution test system [47] which includes a 2feeder substation, 32 buses, 5 looping branches, 5 tie switches and 32 sectionalizing switches. This system initially has a substation with capacity of $2600 \mathrm{~kW}$ which can be upgraded to $4355 \mathrm{~kW}$. Also, it contains 15 upgradable existing branch sections, and 12 routes for installing new branch sections, which have been tabulated in Table 1. The expansion planning horizon consists of 3 stages while 3 new load points will be installed in the third stage. These new load points have been shown in Table 2, and complete data can be obtained from [47]. To have better insight about the test system, the initial structure of the distribution network has been shown in Fig. 4. Also, Fig. 5 shows active power demand in each stage considering 3 load levels (peak load, medium load and low load) graphically.

In the following, some discussions have been presented on the proposed framework.

\section{A. Solution Algorithm Performance Analysis}

To better illustrate the efficiency of the proposed framework, two scenarios have been studied and discussed below.

\section{Scenario 1: Single objective MDEP problem}

In this case, the MPSO algorithm has been employed for single objective MDEP problem considering DGs. About the MPSO algorithm, the adjusting parameters include number of initial population, number 
of iterations, mutation constant $(\psi)$ and learning factors $\left(c_{1}, c_{2}\right.$, and $\left.\omega\right)$. These parameters are: number of population $=200$, number of iterations $=100, c_{1}=2, c_{2}=2, \psi=2$, and $\omega=0.4 \sim 0.9$. To have better illustration of the performance of the MPSO algorithm, its results have been compared with original PSO and GA. All the defined objective functions have been individually studied. It is noted that to evaluate the performance of the proposed algorithm, the optimization problem has been solved for 10 trials and the best solution, worst solution, average of the solutions (for 10 trials), and the standard deviations obtained by optimizing the all objective functions using original PSO, GA and MPSO separately have been shown in Tables 3 to 6, respectively. Tables 3 to 6 illustrate that the MPSO algorithm is capable of finding the better solutions with respect to the original PSO and GA algorithms. Also, the performance of the MPSO solution is more robust than the other algorithms while the standard deviation for this algorithm is lower than others. That is, in different trials, the MPSO will approximately reveal the same results.

Single line structure of the distribution network in all stages has been shown in Fig. 6 while the cost function has been selected as the objective function. In this state, the END, active power losses and VSI values are $48527(\mathrm{kWh} /$ year), $432.5161(\mathrm{~kW})$ and 0.009 , respectively. It is noted that there is no rewiring and DG units' installation in this case.

\section{Scenario 2: Multiobjective MDEP problem}

In this case, all the objective functions are being simultaneously minimized using modified multiobjective PSO algorithm. Generally, the cost function conflicts with the functions of END and active power losses. However, the END function and the function of active power losses are not in conflict. Also, according to (18) to (20), it is clear that when DG output is decreased, VSI is increased (in this study a DG output is modeled by a negative load). Thus, VSI nearly is in conflict with END and active power losses. To support the above statements regarding the relationships between the objective functions, a set of nondominated solutions using (32) have been obtained. Fig. 7 shows the Pareto front of the two-objective MDEP optimization problem including: I\&O cost vs. active power losses and END vs. VSI. Moreover, Fig. 8 shows a set of non-dominated solutions of three-objective cases. From these figures, it can be 
concluded that the END and power losses are in conflict with the I\&O cost. Finally, a set of nondominated solutions which are trade-off between all objective functions are tabulated in Table 7. As it can be seen from this table, cases I to IV refer to single objective cases and the other cases relate to multiobjective cases with different weighting factors for the objective functions.

In the fuzzy decision making approach, the importance of each objective function is determined by the weighting coefficients such that $\sum_{i=1}^{N_{\text {obj }}} w_{i}=1$. Table 7 shows the results of implementing the fuzzy decision making approach over Pareto optimal set. To have more comparison between single objective and multiobjective problem, cases I to IV are devoted to the single objective optimization results.

By analyzing the results of Table 7, the following observations can be concluded:

- The objectives $F_{2}$ and $F_{3}$ have the same behavior approximately. The reason for this claim can be inferred from the results of cases I, III and VII of Table 7. In cases I and III when each of $F_{2}$ or $F_{3}$ is minimized individuality; the other is also being close to the minimized value. In case VII, different coefficients for $F_{2}$ and $F_{3}$ do not change the obtained solutions significantly. That is these objective functions are similar.

- The objectives $F_{1}$ and $F_{2}$ are conflicting objective functions. Indeed, while the network output of DG units are reduced, the I\&O cost has been decreased but END has been increased, simultaneously. Furthermore, cases I, II, V-2, V-3, VIII-2 and VIII-3 prove this statement.

- The objectives $F_{1}$ and $F_{3}$ are conflicting objective functions. Indeed, while the network output of DG units are increased, the I\&O cost has been increased but power losses have been decreased, simultaneously. Furthermore, cases I, III, V-2 and V-4 prove this statement.

- The objective $F_{2}$ is in contrast with respect to the objective $F_{4}$. To minimize the END, DG units should generate more active power, so the VSI will be increased. Cases II, IV, VIII-3 and VIII-4 show this matter. 
- The voltage stability index has a small value in all cases; therefore case V-1 has a suitable trade-off between all objective functions.

The above observations have been resulted from single objective and three-dimensional optimization problem. Similar results can be concluded from the case IX as explained below:

- Results of case IX-3 is similar to case IX-4. Despite the low value of importance for each of these objective functions in these two cases, the results have not been changed. That is $F_{2}$ and $F_{3}$ have the same behavior.

- In case IX-2, worsening the condition of the $F_{1}$, has improved the condition of $F_{2}$ and $F_{3}$. This matter confirms that $F_{1}$ has a conflict with $F_{2}$ and $F_{3}$.

- Case IX-5 is similar to case IX-1. In this case, low preference of $F_{4}\left(w_{4}=0.1\right)$ doesn't affect the compromise between objective functions. In other words, the variation of $F_{4}$ does not have significant influence.

- According to the results of case IV in Table 7, it is clear that the voltage stability index has a small value which indicates high voltage stability margin. That is, the short circuit current of all buses are under the capacity of breakers. Therefore, the short circuit current as an index is not considered in the rest of the case studies, so the best compromise is evaluated when assuming the same importance for the objective functions, i.e., $w_{1}=w_{2}=w_{3}=0.33$ and $w_{4}=0$ in Eq. (34). Fig. 9 shows a single line diagram of distribution system in all stages. For instance, in third stage, branch ${ }_{3-23}$ (that means the branch between buses 3 and 23), branch $_{6-7}$, branch $_{12-13}$, branch $_{16-17}$, branch $_{30-31}$, and branch $_{31-32}$ are required to be rewired, and switch 8 -21 (that means the switch located at line between buses 8 and 21), switch $_{8-9}$, switch $28-29$, switch $_{10-15}$, and switch $_{15-16}$ should be opened because the structure of the distribution network should be radial. The outputs of DGs in all stages have been tabulated in Table 8. Figs. 10 to 12 illustrate the values of objective functions which obtained in different cases (single objective, and multiobjective cases). As it can be seen from these figures, the best compromised solution of the case with $\mathrm{w}_{1}=\mathrm{w}_{2}=\mathrm{w}_{3}=0.33$ and $\mathrm{w}_{4}=0$ approximately has the value near to the 
mean value of other cases (Case I to III in Table 7). Furthermore, it can be inferred from these figures that END and active power losses are not in conflict due to the fact that to improve END value, DG output values should be increased, and accordingly the active power losses will be reduced. This matter can be concluded from the results.

\section{B. Equivalent cost of reliability and loss}

According to the first and second rows of table 7, the cost value of cases I and II are $1020303 \mathrm{R}$ and $12432000 \mathrm{R}$, respectively. Indeed, the cases I and II refer to the minimization of cost and ENS as a reliability index, respectively. It can inferred from these results that improving reliability of the distribution network costs $12432000-1020303=11411697 \mathrm{R} \$$. This difference can be nominated as the equivalent cost of reliability. That is, the difference of cost function in cases I and II comes from installing new capacity of DG units and line rewiring to enhance reliability of system. Similarly, by comparing the cost values for cases I and III (minimizing loss function), the cost of loss can be determined. That is, the difference between these two costs (the minimum cost and cost in the case of optimal electrical loss) which is equal to $11953000 \mathrm{R} \$$ can be considered as the equivalent cost of the power loss improvement.

\section{Effect of reliability parameters}

This section aims to investigate the impact of the reliability parameters on the objective function values. As mentioned earlier, the reliability parameters include the annual failure rate $(\beta)$, the reparation time $(t)$ and the restoration time ( $\left.\mathrm{t}^{\prime}\right)$. According to the equation (11), ( $U=\beta \times t$ and $\left.U^{\prime}=\beta \times t^{\prime}\right)$, when $U$ and $U^{\prime}$ is changed (by change of the annual failure rate or the reparation time and the restoration time) the objective function values will be changed, too. To assess the effect of reliability parameters on the results of MDEP, the optimization problem has been studied with different values of $U$ and $U^{\prime}$. Indeed, the nominal values of $U$ and $U^{\prime}$ have been multiplied with the factors $0.6,0.8,0.9,1,1.1,1.2$ and 1.4 for the 
case of multiobjective optimization problem with equal importance values of the objective functions $\left(w_{1}=\right.$ $\left.w_{2}=w_{3}=0.33\right)$. The simulation results of this case have been illustrated in figures 13,14 and 15 . It can be seen in the figures 13 and 14, the ENS and cost values are directly related to the $U$ and $U^{\prime}$ factors. According to the Eq. (15), power loss and $U$ and $U^{\prime}$ are not related to each other. However, because the objective functions have been optimized simultaneously with equal importance factor, fluctuations in the objective functions of cost and power losses are justifiable. Finally, the objective function values, capacity installation of DG units and the number of rewiring lines for each $U$ and $U$ ' factors are demonstrated in the Table 9.

\section{Effect of load changes}

In this section to evaluate the sensitivity of the objective functions to load variations, coefficients for the load $(0.8,0.9,1,1.05$, and 1.1$)$ are considered. Accordingly, the multiobjective DEP problem with equal importance factors of the objective functions $\left(w_{1}=w_{2}=w_{3}=0.33\right)$ for different load coefficients is solved. The simulation results have been shown in Figures 16, 17 and 18 graphically. As it can be seen in Figures 16 and 17, cost values and ENS are directly related to the changes in load. In other words, the increment of load increases the ENS and cost values. The power loss does not have specific variations. Firstly, it seems by increasing the load, the power loss should be increased. However, by increasing the load values, the network expansion planning will be changed. Indeed, the network needs to be reinforced by installing new DG and feeder rewiring. Generally, the change in installation of DG units is proportional to the load variations. Accordingly, adding new capacities of DGs will increase the cost function. The objective function values, installation capacity of DGs and the number of rewiring lines by different coefficients of load are tabulated in the Table 10. It can be seen from this table that by increasing the load, the number of rewiring or capacity installations will be increased. For instance, by changing load factor 1 to 1.05 , the number of rewiring has been decreased while the capacity installations have been significantly increased. Another observation shows that for the load factor 1.1 in comparison with load 
factor 1.05, although the capacity installations are decreased, however, the number of rewiring is increased.

\section{E. Evaluating the multiobjective optimization performance}

In this section, the obtained Pareto solutions by MPSO have been compared with the other original optimization algorithms (here is PSO and GA algorithm) using presented metrics in subsection III. D. Obtained GD, SP and D metrics for the MPSO, PSO and GA for the two-objective optimization cases and all three-objective functions have been tabulated in Table 11. From this table, it is clear that the proposed algorithm can obtain better Pareto front with respect to the other algorithms. This is because the most values of SP and GD of the MPSO algorithm are lower than those obtained by other original algorithms. Also, the most values of D metric of the proposed algorithm is greater than those obtained by other original algorithms which implies the efficiency of the obtained Pareto front by the proposed MPSO algorithm.

\section{Conclusions}

In this paper, to enhance the reliability and security levels of power distribution networks, the reliability index (i.e. END) and security index (i.e. voltage stability index based on the SCC) have been included in the MDEP problem. Accordingly, in the proposed MDEP framework, I\&O cost, END, active power losses and VSI have been selected as objective functions to be optimized, simultaneously. Accordingly, a set of non-dominated solution is considered as the solutions of the MDEP problem. A fuzzy decision making has been used to trade-off between obtained Pareto solutions. Moreover, multiobjective MPSO optimization technique has been proposed to solve the MDEP problem. The proposed method can compromise the conflicting objectives of the MDEP problem in such a way that the system planner's concerns about the reliability and security of the distribution networks are relieved with a tolerable and reasonable cost. Also, this paper has shown the effectiveness of the proposed MPSO which efficiently 
generates optimal Pareto solutions. Therefore, it can be inferred that the proposed multiobjective approach can lead to a more efficient utilization of planning options (rewiring, adding feeders, status of switches, and placement and sizing of DG units) and it permits the system planners to estimate how likely the system to be expanded and what are the possible actions for the future system structure in the presence of DG units.

The research work is under way in order to simulate DGs and load uncertainty in the stochastic multiobjective framework for DEP problem.

\section{References}

[1] Boutsika Th., Papathanassiou S., Drossos N.:'Calculation of the fault level contribution of distributed generation according to IEC Standard 60909’, Proceedings of CIGRE Symposium Power Systems with Dispersed Generation, Athens, 2005

[2] Boulaxis NG., Papadopoulos MP.:'Optimal feeder routing in distribution system planning using dynamic programming technique and GIS facilities', IEEE Trans Power Deliv. 2002, 17, (1), pp. 242-247

[3] Ponnavaikko M., Prakasa Rao KS.:‘An approach to optimal distribution system planning through conductor gradation', IEEE Trans Power Appl Syst. 1982, 101, (6), pp. 1735-1342

[4] Aoki K., Nara K., Satoh T., Kitagawa M., Yamanaka K.:‘New approximate optimization method for distribution system planning', IEEE Trans Power Syst. 1990, 5, (1), pp. 113-126

[5] Fawzi TH., Ali KF., El-Sobki SM.: ‘A new planning model for distribution systems’, IEEE Trans Power Appl Syst. 1983, 102, (9), pp. 3010-3017

[6] Temraz HK., Salama MMA.: 'A planning model for siting, sizing and timing of distribution substations and defining the associated service area', Electr Power Syst Res. 2002, 62, (2), pp. $17-29$

[7] Miranda V., Ranito JV., Proenca LM.: 'Genetic algorithms in optimal multistage distribution network planning', IEEE Trans Power Syst. 1994, 9, (4), pp. 1927-1933 
[8] Vaziri M., Tomsovic K., Bose A.: 'A directed graph formulation of the multistage distribution expansion problem', IEEE Trans Power Deliv.2004, 19, (3), pp. 1335-1341

[9] Haffner S., Pereira L.F.A., Pereira L.A., Barreto L.S.: 'Multistage model for distribution expansion planning with distributed generation. Part II: Problem formulation', IEEE Trans Power Deliv.2008, 23, (2),pp. 915-923

[10] Haffner S., Pereira L.F.A., Pereira L.A., Barreto L.S.: 'Multistage model for distribution expansion planning with distributed generation. Part II: Numerical results', IEEE Trans Power Deliv. 2008, 23, (2), pp. 924-929

[11] El-Kady MA.: 'Computer-aided planning of distribution substation and primary feeders', IEEE Trans PAS.1984, 103, (6),pp. 1183-1189

[12] GonenT., Foote B.L.: 'Mathematical dynamic optimization model for electrical distribution system planning', Elect. Power Energy Syst.1982, 4, (2), pp. 129-136

[13] Miranda V., Proenca LM: ‘A General methodology for distribution planning under uncertainty, including genetic algorithms and fuzzy models in a multicriteria environment', Proc Stockholm Power Tech SPT 95.1995, Stockholm, Sweden, pp. 832-837

[14] Neiname V.: 'On development planning of electricity distribution networks' 2001, Ph. D. Dissertation, Royal Institute of Technology, Stockholm, Sweden.

[15] Ganguly S., Sahoo N.C., Das D.: 'Mono- and multiobjective planning of electrical distribution networks using particle swarm optimization’, Applied Soft Computing.2011, 11, pp. 2391-2405

[16] Niknam T., Taheri S. I., Aghaei J., Tabatabaei S., Nayeripour M.: 'A modified honey bee mating optimization algorithm for multiobjective placement of renewable energy resources', Applied Energy. 2011,88, (12), pp. 4817-30

[17] Popovi ZN, Popovi DS: 'Graph theory based formulation of multi-period distribution expansion problems', Electric Power Systems Research.2010, 80, (10), pp. 1256-1266 
[18] Falaghi H., Singh C., Haghifam M.-R., Ramezani M.: 'DG integrated multistage distribution system expansion planning', International Journal of Electrical Power and Energy Systems. 2011, 33, (8), pp. 1489-1497

[19] Ouyang W., Cheng H., Zhang X., Yao L.: 'Distribution network planning method considering distributed generation for peak cutting', Energy Conversion and Management, 2010, 51, (12), pp. 2394-2401

[20] Soroudi A., Ehsan M.: 'A distribution network expansion planning model considering distributed generation options and techno-economical issues’, Energy, 2010, 35, (8), pp. 33643374

[21] Vidyasagar E., Prasad PVN, Fatima A.: 'Reliability Improvement of a Radial Feeder Using Multiple Fault Passage Indicators’, Energy Procedia, 2012, 14, pp. 223-228

[22] Hamedi H, Gandomkar M: ‘A straightforward approach to minimizing unsupplied energy and power loss through DG placement and evaluating power quality in relation to load variations over time’, International Journal of Electrical Power \& Energy Systems, 2012, 35, (1), pp. 9396

[23] Zhang P., Li W., Wang S.:'Reliability-oriented distribution network reconfiguration considering uncertainties of data by interval analysis', International Journal of Electrical Power \& Energy Systems. 2012, 34, (1), pp. 138-144

[24] Boutsika TN, Papathanassiou SA: 'Short-circuit calculations in networks with distributed generation’, Electric Power Systems Research.2008, 78, (7),pp.1181-1191

[25] Papathanassiou SA.: 'A technical evaluation framework for the connection of DG to the distribution network', Electric Power Systems Research.2007, 77, (1), pp. 24-34

[26] Koutroumpezis GN, Safigianni AS.: 'Optimum allocation of the maximum possible distributed generation penetration in a distribution network', Electric Power Systems Research,2010, 80, (12),pp. 1421-1427 
[27] Malekpour AR, Niknam T, Pahwa A, Kavousifard A.: 'Multi-objective Stochastic Distribution Feeder Reconfiguration in Systems with Wind Power Generators and Fuel Cells Using Point Estimate Method', IEEE Trans Power Syst. 2013, 28, (2), pp. 1483-1492.

[28] Niknam T, Kavousifard A, Aghaei J.: 'Scenario-Based Multi-objective Distribution Feeder Reconfiguration considering Wind Power Using Adaptive Modified PSO', IET Renewable Power Generation, 2012, 6, (4), pp. 236 - 247.

[29] Niknam T, Kavousifard A.: 'Impact of Thermal Recovery and Hydrogen Production of Fuel Cell Power Plants on Distribution Feeder Reconfiguration’, IET Generation, Transmission \& Distribution, 2012, 6, (9), pp. 831 - 843.

[30] Niknam T, Kavousifard A.: 'Multi-objective Stochastic Distribution Feeder Reconfiguration Problem considering Hydrogen and Thermal Energy Production by PEM Fuel Cell Power Plant’, Energy, 2012, 42, (1), pp. 563-573.

[31] Niknam T, Azad farsani E, and Jabbari M.: ‘A New hybrid Evolutionary Algorithm Based on new fuzzy adaptive PSO and NM algorithms for Distribution Feeder Reconfiguration', Energy Conversion and Management, 2012, 54, (1), pp. 7-16.

[32] Niknam T.: 'An efficient multi-objective HBMO algorithm for distribution feeder reconfiguration', Expert Systems with Applications, 2011, 38, 3, pp. 2878-2887

[33] Billinton R., Allan R N.: 'Reliability Evaluation of Power Systems’, New York and London, 1996,2th ed. Reference to a chapter in an edited book

[34] Khalesi N, Rezaei N, Haghifam MR.:'DG allocation with application of dynamic programming for loss reduction and reliability improvement', International Journal of Electrical Power and Energy Syst. 2011,33, (2), pp. 288-95

[35] Deng G.: 'Research on voltage stability of power system based on shortcircuit capacity', 2009, Ph.D. dissertation, Wuhan Univ.

[36] Deng G., Sun Y., Xu J: ‘A New Index of Voltage Stability ConsideringDistribution Network’, IEEE Asia Conf., Pacific Power and Energy Engine., 2009, pp. 1-4 
[37] Taylor C. W.:'Power System Voltage Stability’, 1994, New York, McGraw-HillEducation.

[38] Huang L, Xu J, Sun Y, Cui T, Dai F.: 'Online monitoring of wide-area voltage stability based on short circuit capacity’, IEEE Asia Conf., PacificPower and Energy Engine., 2011, pp. 1-5

[39] Abdelaziz AY, Mohammed FM, Mekhamer SF, Badr MAL.: 'Distribution Systems Reconfiguration using a modified particle swarm optimization algorithm', Electric Power Systems Research, 2001, 79, (11), pp. 1521-1530

[40] Kennedy J., Eberhart R.: 'Particle swarm optimization', IEEE international conference on neural networks, Piscataway, 1995, NJ, 4, pp. 1942-1948

[41] Wong K. P., Dong Z. Y.:'Differential evolution, an alternative approach to evolutionary algorithm', Proceedings of the 13th International Conference on Intelligent Systems Application to Power Systems, 2005, pp. 73-83.

[42] Niknam T, Narimani MR, Jabbari M, Malekpour AR.: 'A modified shuffle frog leaping algorithm for multiobjective optimal power flow', Energy, 2011, 36, (11), pp. 6420-6432

[43] Tan CH., Goh CK., Tan KC., Tay A.:'A cooperative co evolutionary algorithm for multiobjective particle swarm optimization', IEEE Congress on Evolutionary Comput., 2007, 25, (28), pp. 3180-3186.

[44] Chen T. H., Chen M. S., Hwang K. J., Kotas P, Chebli EA.: 'Distribution System Power Flow Analysis - A Rigid Approach', IEEE Trans on Power Deliv.1991,6, (3), pp. 1146-1152

[45] Schott, J. R., Fault tolerant design using single and multicriteria genetic algorithms optimization. Master's thesis, Department of Aeronautics andAstronautics, Massachusetts Institute of Technology, Cambridge, MA, 1995.

[46] Van Veldhuizen DA, Lamont, GB.: 'Multiobjective evolutionary algorithm research: a history and analysis’. Technical report TR-98-03, Dept. Elec. Comput. Eng., Graduate School of Eng., Air Force Inst. Technol., Wright-Patterson AFB, OH, USA, 1998.

[47] Baran ME., Wu FF.:'Network reconfiguration in distribution systems for loss reduction and load balancing', IEEE Trans Power Deliv. 1989, 4, (2), pp. 1401-1407 


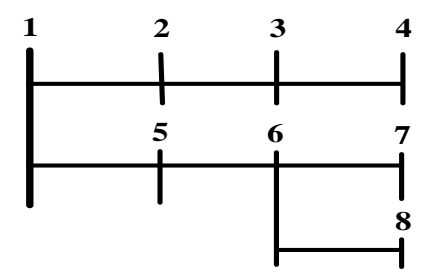

Fig. 1. Simple single line distribution network

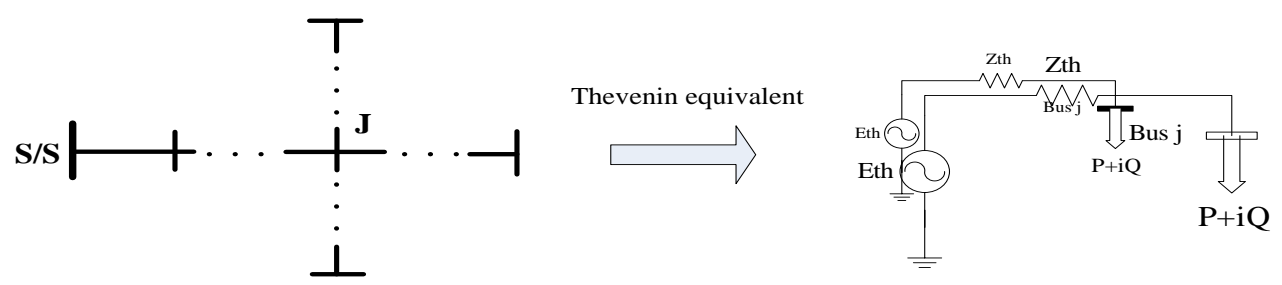

Fig. 2. Thevenin equivalent system of bus $j$ 


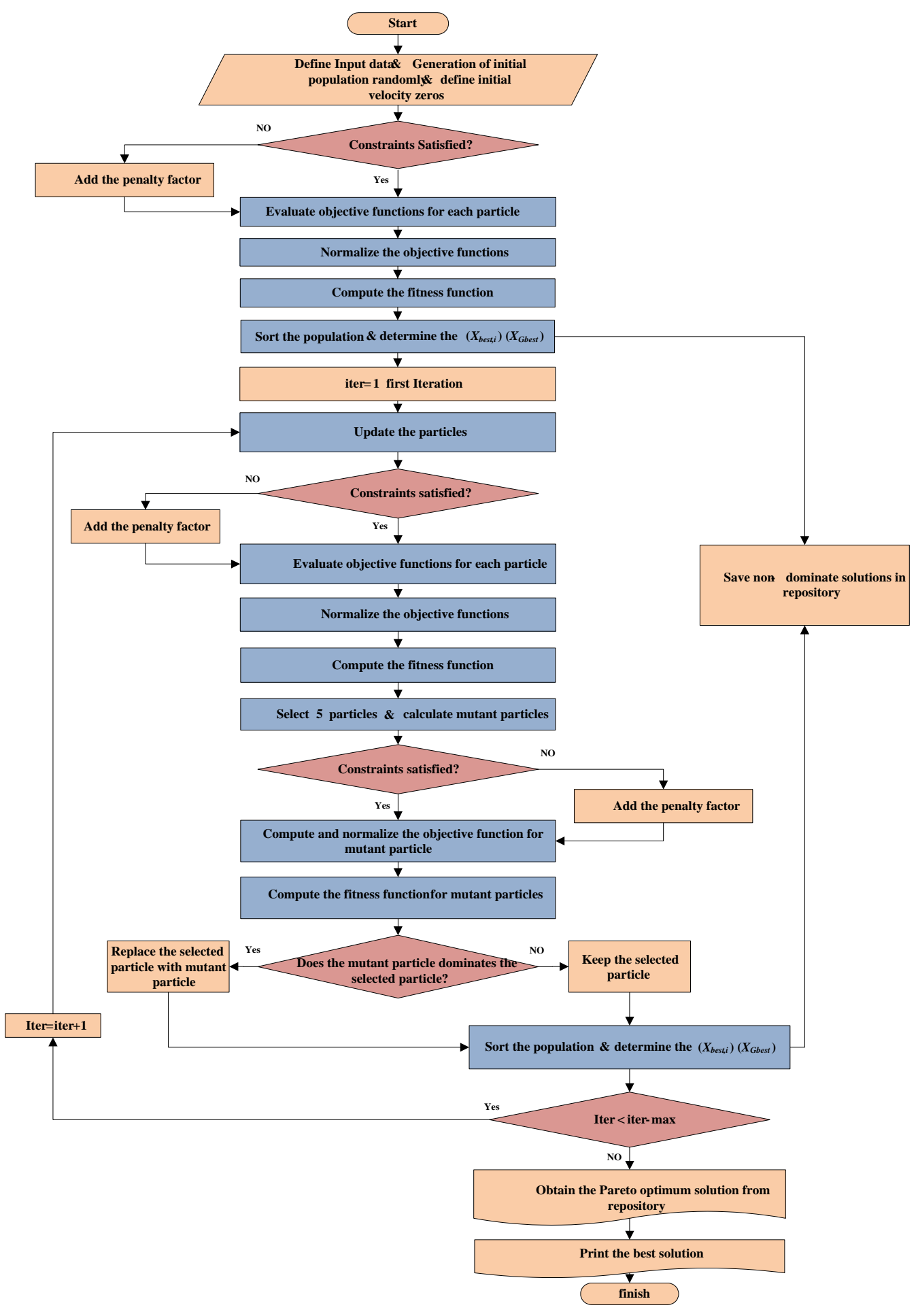

Fig. 3. A flowchart of the MPSO algorithm for solving MDEP problem 


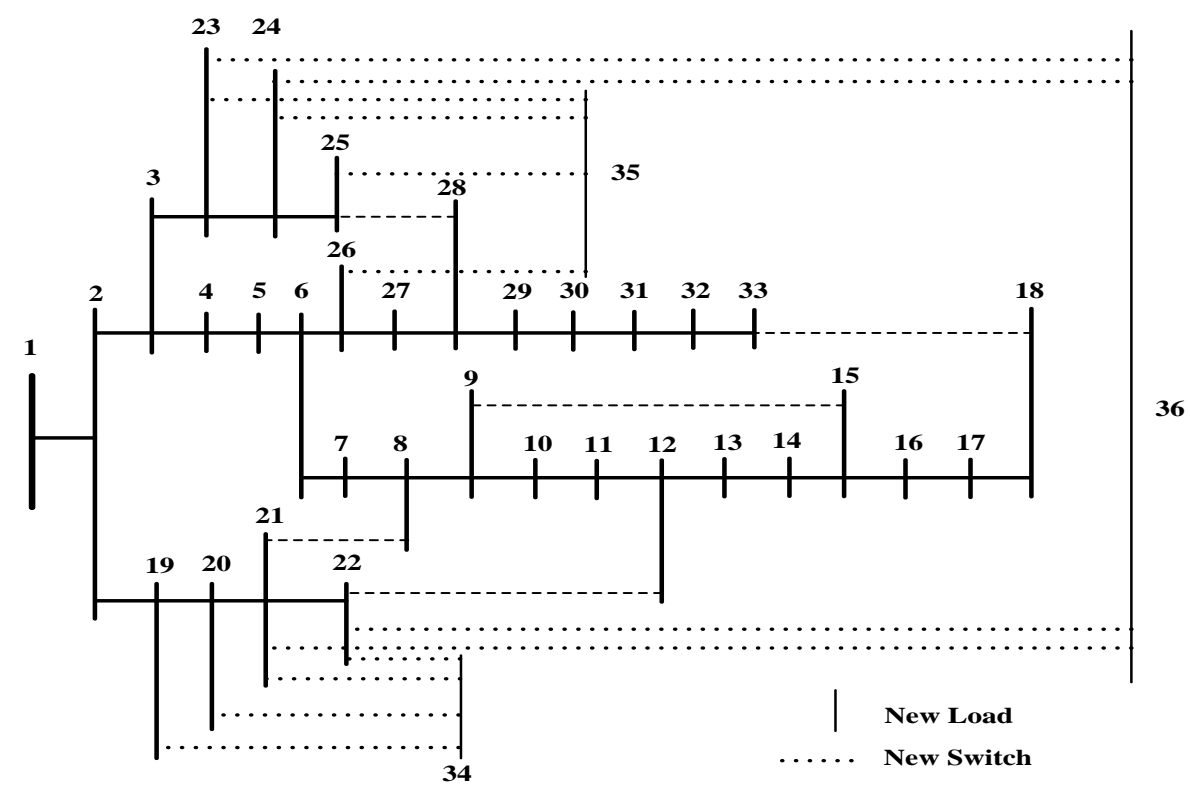

Fig. 4. A single line diagram of the distribution test system

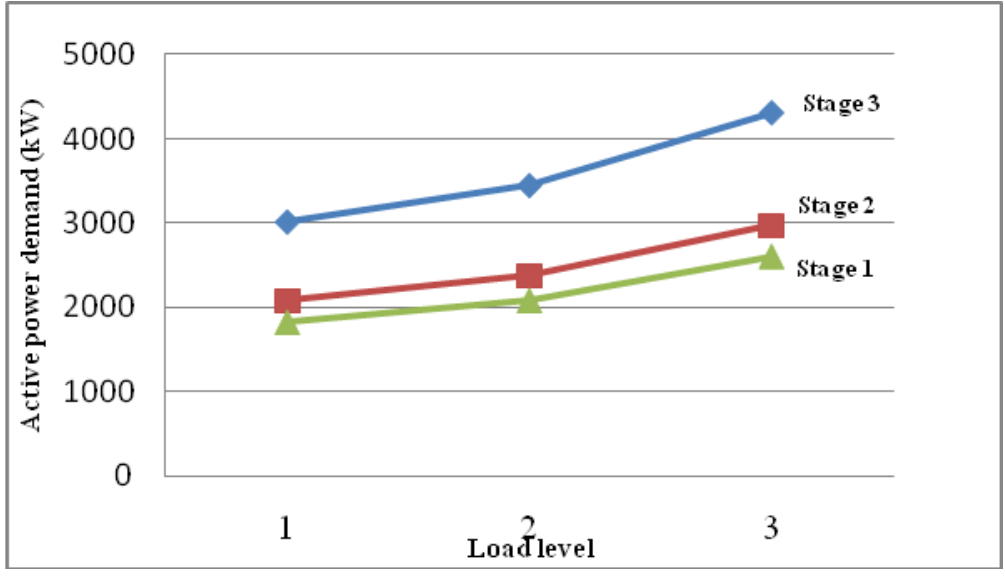

Fig. 5. Total demand of all stages in each load level 


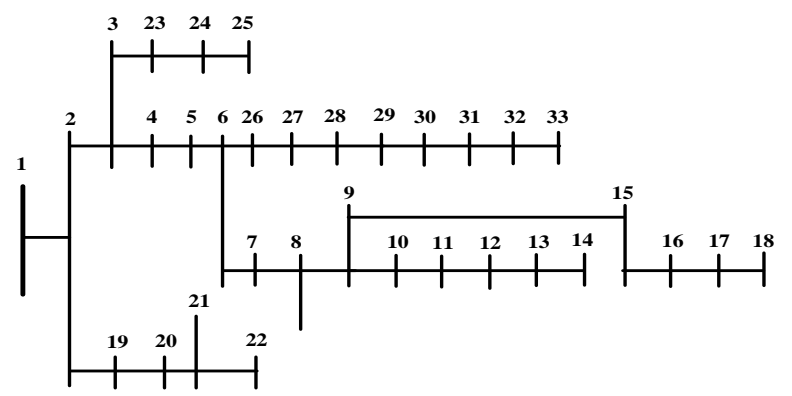

Stage $1 \& 2$

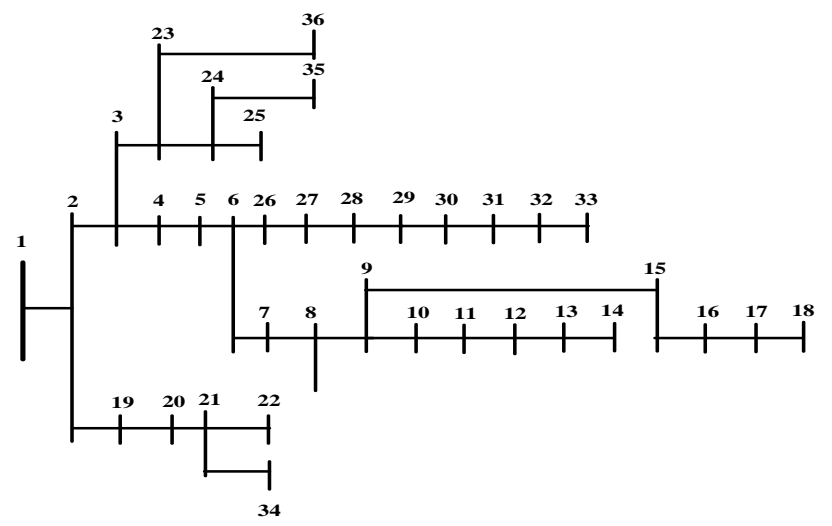

Stage 3

Fig. 6. Single diagram of the distribution network for optimum I\&O cost in three stages 

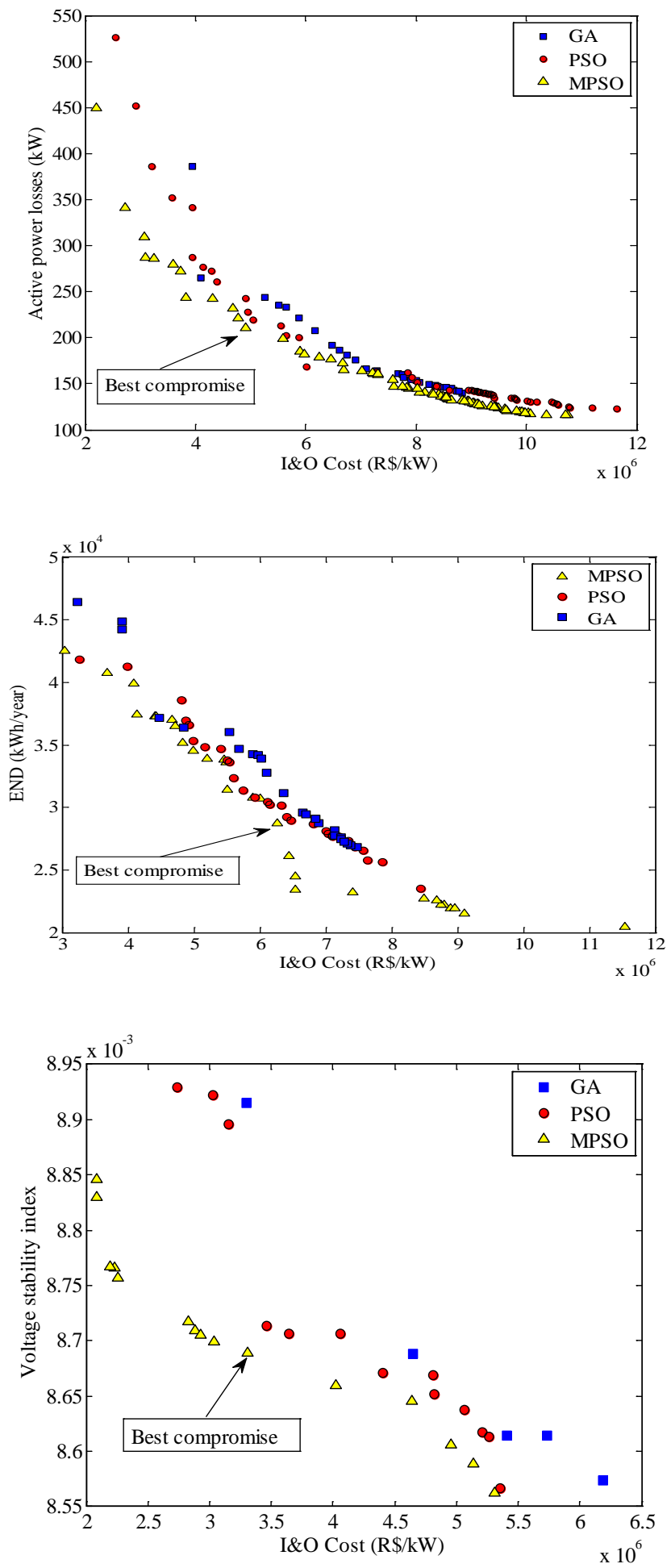

Fig. 7. Two-dimensional Pareto fronts 

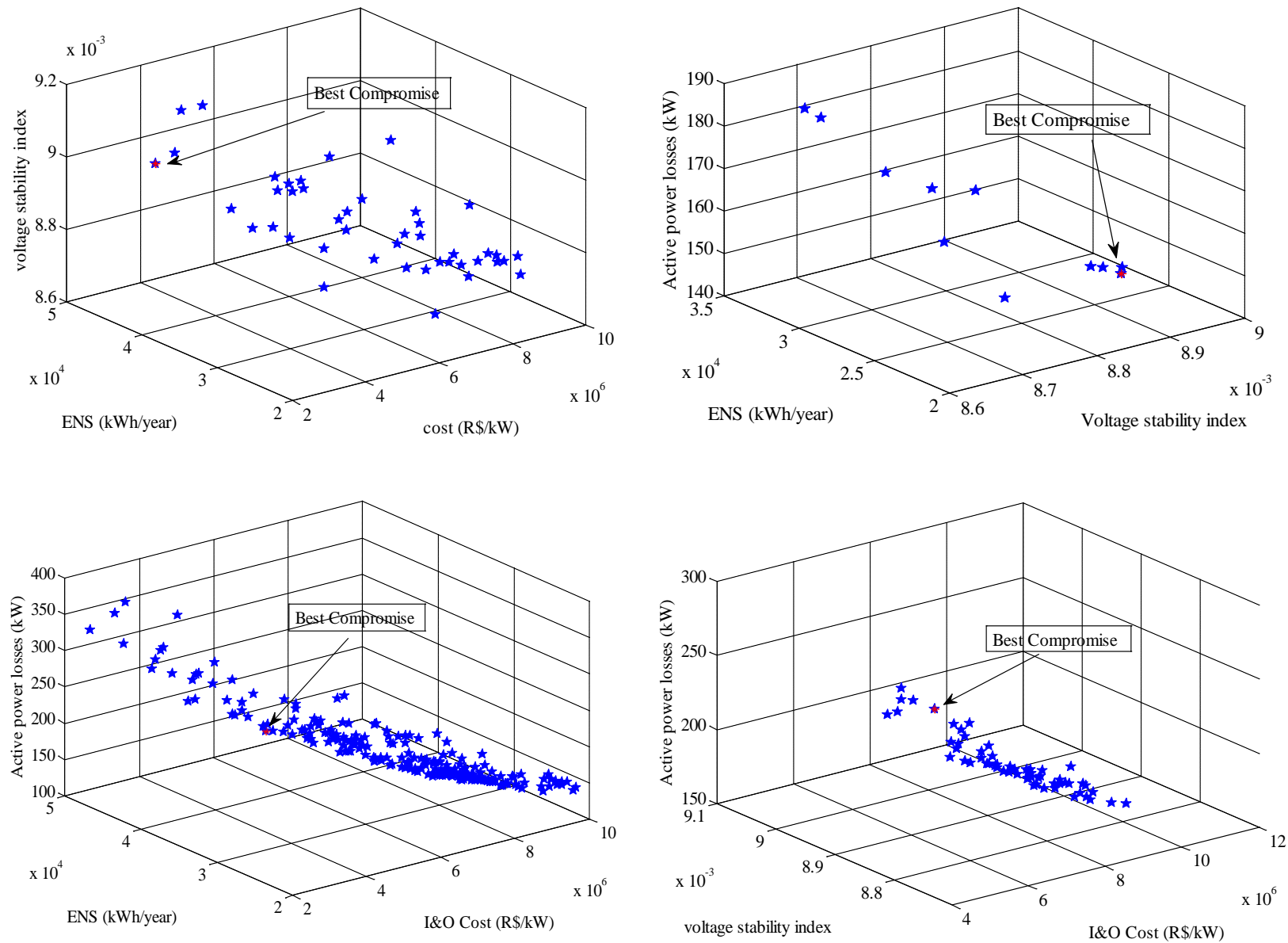

Fig. 8.Three-dimensional Pareto fronts for different combinations of objective functions 


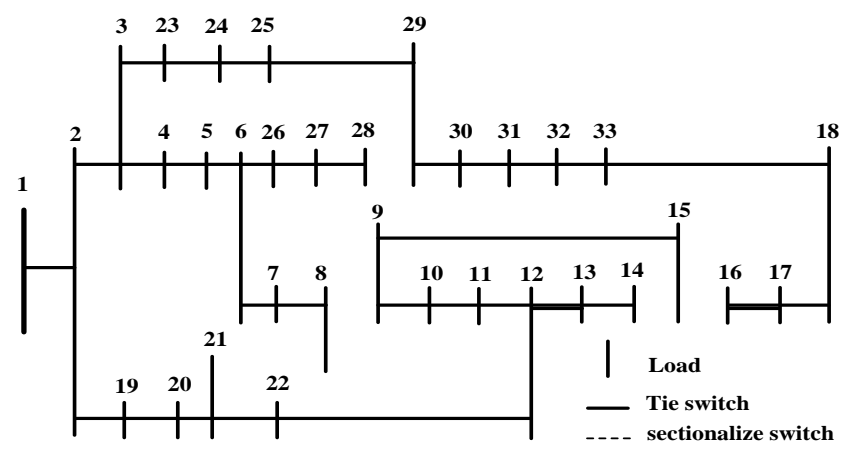

Stage 1

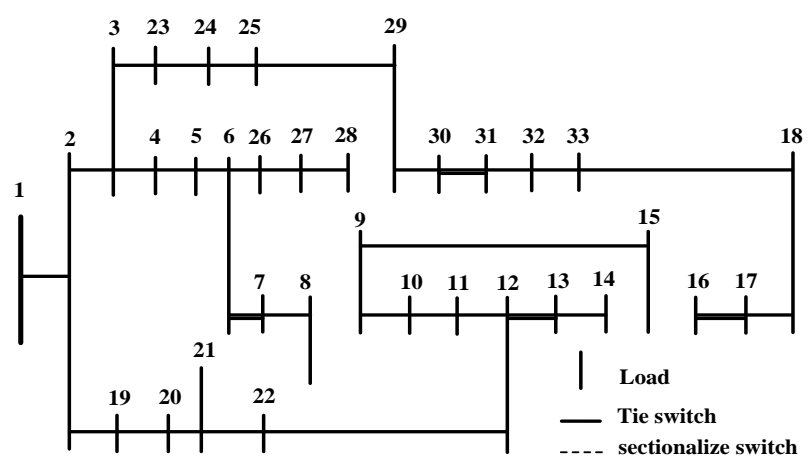

Stage 2

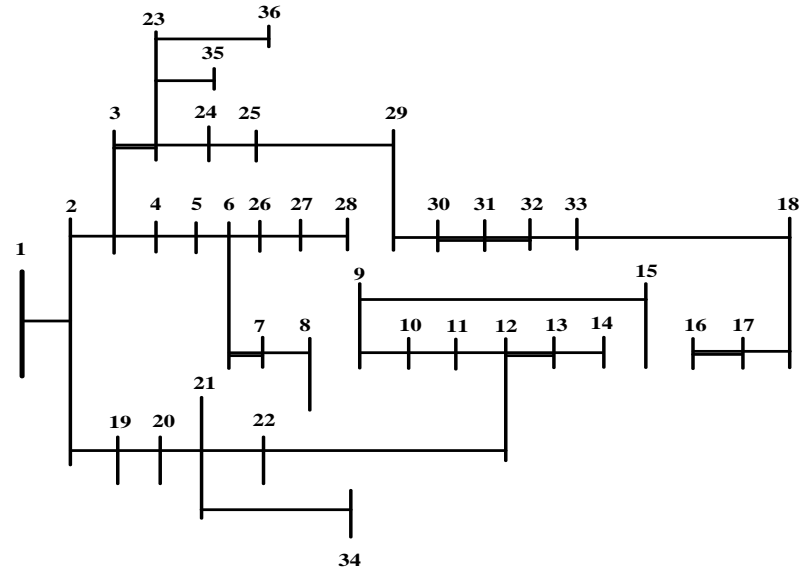

Stage 3

Fig. 9. Single diagram of the distribution network for the best compromised solution in the case of $w_{1}=w_{2}=w_{3}=0.33$ and $w_{4}=0$ 


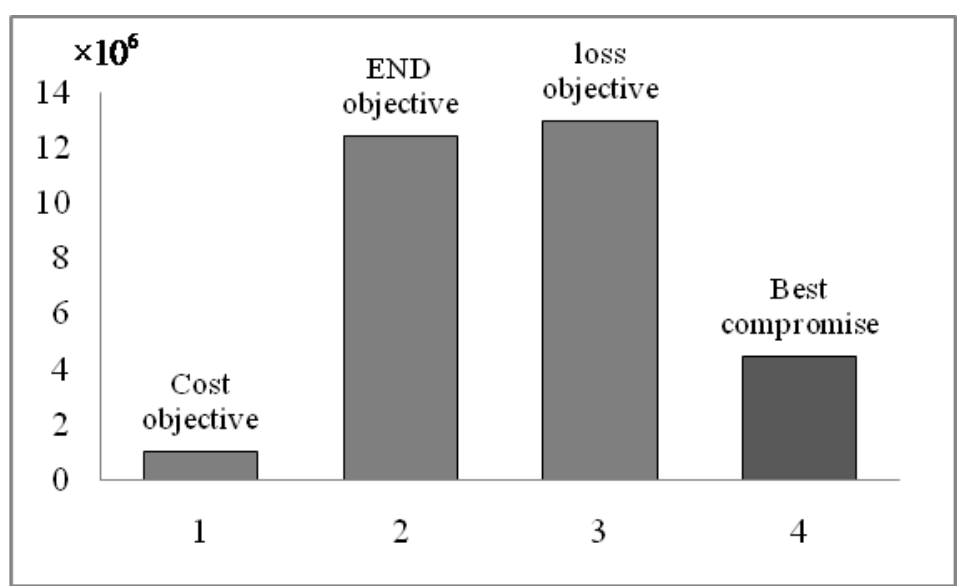

Fig. 10.Comparing cost value in the best compromised solution with optimum cost, optimum END, and optimum active power losses

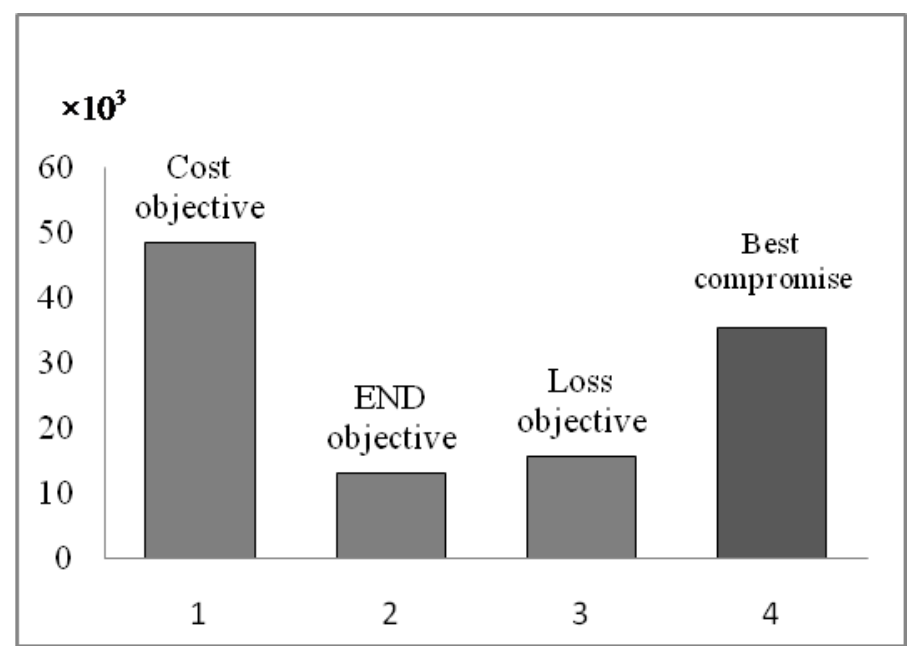

Fig. 11.Comparing END value in the best compromised solution with optimum cost, optimum END, and optimum active power losses 


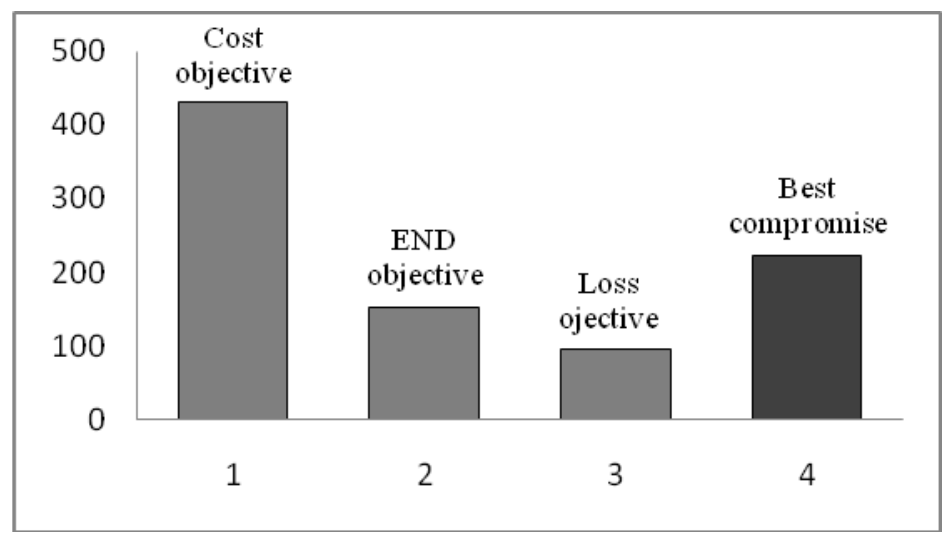

Fig. 12. Comparing active power losses value in the best compromised solution with optimum cost, Optimum END, and optimum active power losses

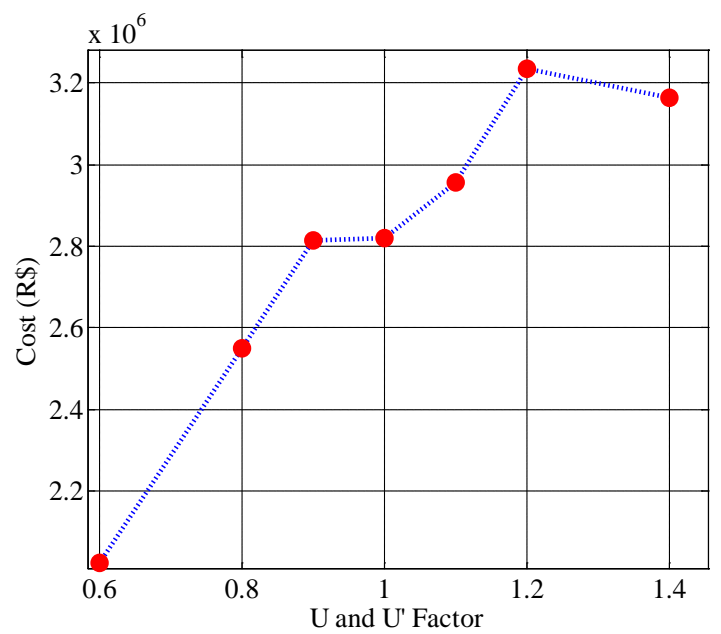

Figure 13: The cost sensitivity with respect to the U and U' changes 


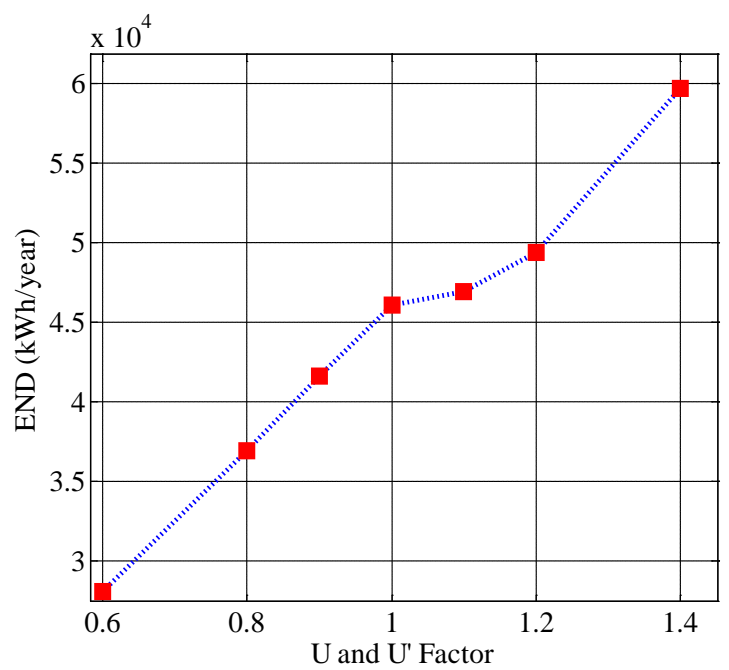

Figure 14: The END sensitivity with respect to the U and U' changes

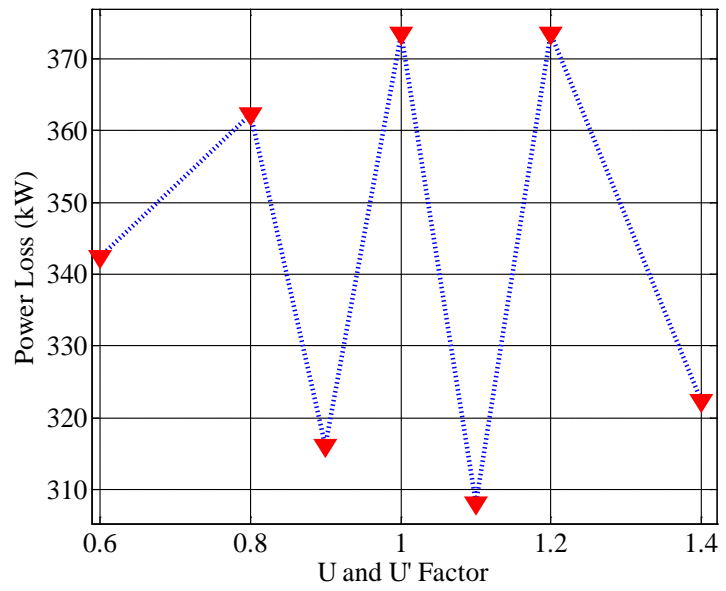

Figure 15: The power loss sensitivity with respect to the $U$ and $U$ ' changes 


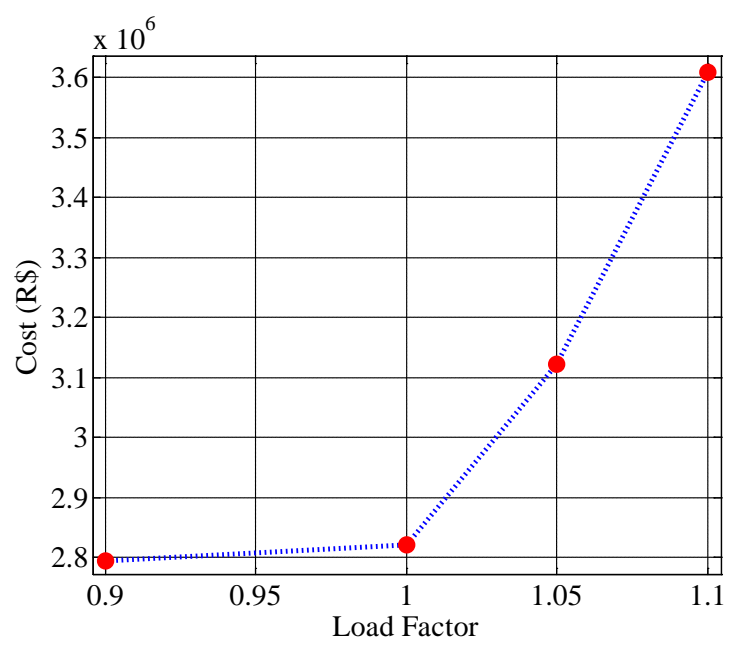

Figure 16: The cost sensitivity with respect to the load changes

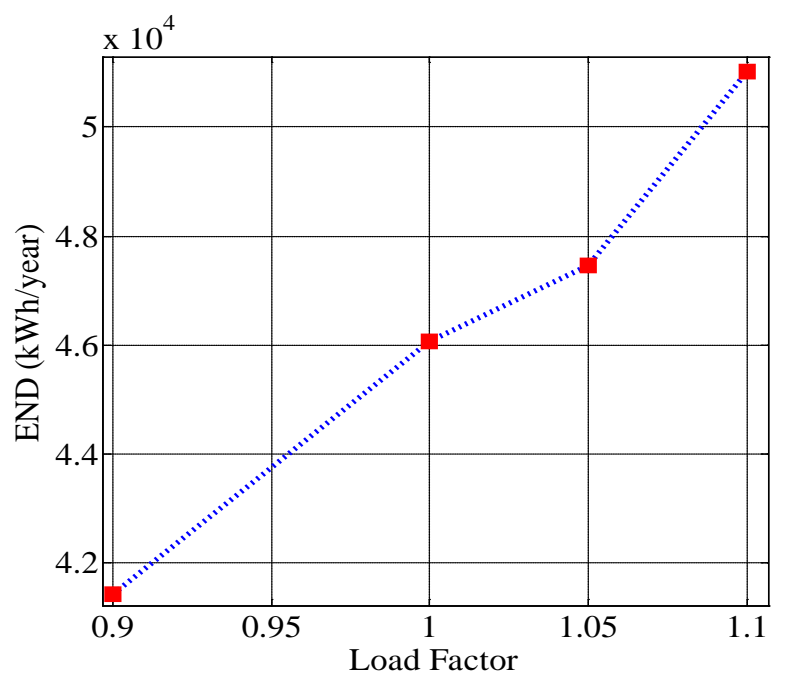

Figure 17: The END sensitivity with respect to the load changes 


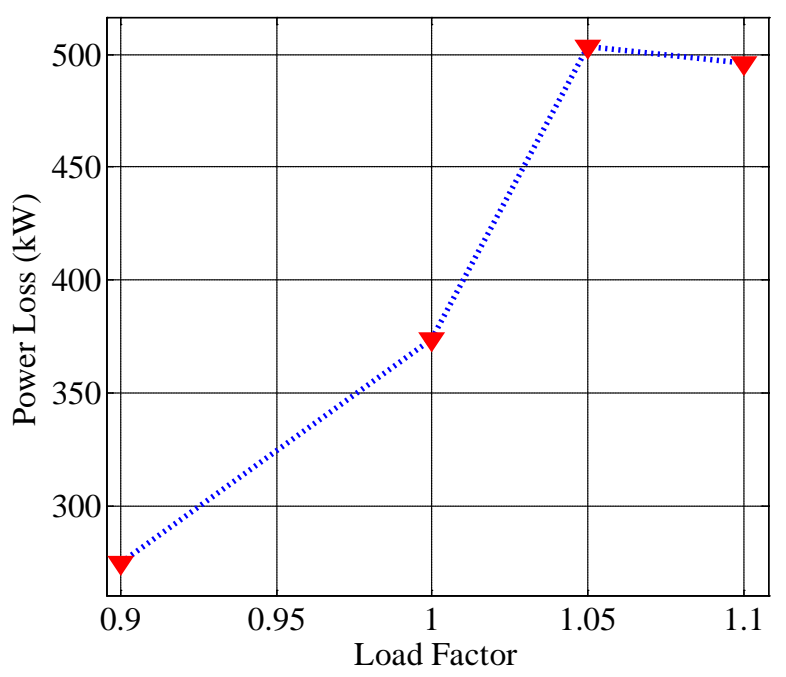

Figure 18: The power loss sensitivity with respect to the load changes 
Tables:

Table 1. Information of new candidate branches

\begin{tabular}{ccccccc}
\hline $\begin{array}{c}\text { No. of new } \\
\text { branch }\end{array}$ & From bus & to bus & $\mathrm{R}(\Omega)$ & $\mathrm{X}(\Omega)$ & $\mathrm{U}(\mathrm{h} / \mathrm{y}$ ear $)$ & U'(h/year) \\
\hline 1 & 19 & 34 & 0.1 & 0.2 & 0.5 & 0.08 \\
2 & 20 & 34 & 0.15 & 0.2 & 0.7 & 0.07 \\
3 & 21 & 34 & 0.1 & 0.3 & 0.9 & 0.05 \\
4 & 22 & 34 & 0.2 & 0.25 & 1 & 0.05 \\
5 & 23 & 35 & 0.1 & 0.2 & 0.6 & 0.02 \\
6 & 24 & 35 & 0.1 & 0.3 & 0.8 & 0.04 \\
7 & 25 & 35 & 0.15 & 0.2 & 0.7 & 0.01 \\
8 & 26 & 35 & 0.2 & 0.25 & 0.1 & 0.05 \\
9 & 21 & 36 & 0.2 & 0.25 & 1 & 0.07 \\
10 & 22 & 36 & 0.1 & 0.2 & 1 & 0.07 \\
11 & 23 & 36 & 0.1 & 0.3 & 1 & 0.04 \\
12 & 24 & 36 & 0.15 & 0.2 & 0.8 & 0.03 \\
\hline
\end{tabular}

Table 2. Demand information for new buses

\begin{tabular}{ccc}
\hline No. new buses & Active power demand (kW) & Reactive power demand (kVAr) \\
\hline 34 & 300 & 250 \\
35 & 100 & 30 \\
36 & 200 & 80 \\
\hline
\end{tabular}


Table3. The results of the single objective DEP problem considering cost (R\$) objective function

\begin{tabular}{ccccc}
\hline Method & Best solution & Average & Worst solution & Standard deviation \\
\hline MPSO & 1020303.2 & 1116752.738 & 1200502.439 & 64312.2661 \\
PSO & 1212103.3 & 1294472.649 & 1404502.784 & 70362.1013 \\
GA & 1416503.28 & 1540167.7296 & 1822503.088 & 114492.76 \\
\hline
\end{tabular}

Table 4. The results of the single objective DEP problem considering END (kWh/year)objective function

\begin{tabular}{ccccc}
\hline Method & Best solution & Average & Worst solution & Standard deviation \\
\hline MPSO & 13290.300 & 13819.153 & 14135.125 & 185.020 \\
PSO & 14147.925 & 14507.230 & 14147.925 & 147.622 \\
GA & 14724.000 & 15296.088 & 17103.400 & 665.674 \\
\hline
\end{tabular}

Table 5.The results of the single objective DEP problem considering power losses $(\mathrm{kW})$ objective function

\begin{tabular}{ccccc}
\hline Method & Best solution & Average & Worst solution & Standard deviation \\
\hline MPSO & 95.49416147 & 98.7114 & 102.2283754 & 1.9629 \\
PSO & 102.4441959 & 105.0464 & 107.9496435 & 1.5921 \\
GA & 107.9618506 & 112.0971 & 120.4459539 & 3.6666 \\
\hline
\end{tabular}

Table. 6. The results of the single objective DEP problem considering voltage stability index objective function

\begin{tabular}{ccccc}
\hline Method & Best solution & Average & Worst solution & Standard deviation \\
\hline MPSO & 0.008465131 & 0.0084855627 & 0.008503787 & 0.000010695 \\
PSO & 0.008504477 & 0.0085251722 & 0.008537356 & 0.00001289 \\
GA & 0.008539638 & 0.0085718247 & 0.008614487 & 0.000028516 \\
\hline
\end{tabular}


Table 7. Objective function values in all cases

\begin{tabular}{|c|c|c|c|c|c|c|c|c|c|}
\hline \multirow{2}{*}{ Cases } & \multirow{2}{*}{$\begin{array}{l}\text { Sub- } \\
\text { case } \\
\text { NO. }\end{array}$} & \multicolumn{4}{|c|}{ Importance Factor } & \multirow{2}{*}{$F_{1}$} & \multirow{2}{*}{$F_{2}$} & \multirow{2}{*}{$F_{3}$} & \multirow{2}{*}{$F_{4}$} \\
\hline & & $w_{1}$ & $w_{2}$ & $w_{3}$ & $w_{4}$ & & & & \\
\hline Case I & & - & - & - & - & 1020303 & 48527 & 432.5161 & 0.009 \\
\hline Case II & & - & - & - & - & 12432000 & 13290.3 & 151.3026 & 0.0088 \\
\hline Case III & & - & - & - & - & 12973000 & 15846 & 95.49416 & 0.0088 \\
\hline Case IV & & - & - & - & - & 7541700 & 30148 & 200.1479 & 0.008496 \\
\hline \multirow{4}{*}{ Case V } & 1 & 0.33 & 0.33 & 0.33 & - & 2820899 & 46063.98 & 373.5442 & - \\
\hline & 2 & 0.2 & 0.4 & 0.4 & - & 2550602 & 46093.13 & 362.3144 & - \\
\hline & 3 & 0.4 & 0.2 & 0.4 & - & 3527097 & 41248.43 & 250.3062 & - \\
\hline & 4 & 0.4 & 0.4 & 0.2 & - & 3163899 & 42670.78 & 322.403 & - \\
\hline \multirow{4}{*}{ Case VI } & 1 & 0.33 & - & 0.33 & 0.33 & 8773060 & - & 174.8745 & 0.009013 \\
\hline & 2 & 0.2 & - & 0.4 & 0.4 & 8773060 & - & 174.8745 & 0.009013 \\
\hline & 3 & 0.4 & - & 0.2 & 0.4 & 8773060 & - & 174.8745 & 0.009013 \\
\hline & 4 & 0.4 & - & 0.4 & 0.2 & 8773060 & - & 174.8745 & 0.009013 \\
\hline \multirow{4}{*}{ Case VII } & 1 & - & 0.33 & 0.33 & 0.33 & - & 31545.28 & 187.5481 & 0.00864 \\
\hline & 2 & - & 0.2 & 0.4 & 0.4 & - & 30746.8 & 186.3287 & 0.008646 \\
\hline & 3 & - & 0.4 & 0.2 & 0.4 & - & 27239.08 & 158.1417 & 0.008741 \\
\hline & 4 & - & 0.4 & 0.4 & 0.2 & - & 31545.28 & 187.5481 & 0.00864 \\
\hline \multirow{4}{*}{ Case VIII } & 1 & 0.33 & 0.33 & - & 0.33 & 3878294 & 43919.7 & - & 0.009134 \\
\hline & 2 & 0.2 & 0.4 & - & 0.4 & 3878294 & 43919.7 & - & 0.009134 \\
\hline & 3 & 0.4 & 0.2 & - & 0.4 & 6927673 & 30970 & - & 0.009091 \\
\hline & 4 & 0.4 & 0.4 & - & 0.2 & 4190294 & 48861.78 & - & 0.008934 \\
\hline \multirow{5}{*}{ Case IX } & 1 & 0.25 & 0.25 & 0.25 & 0.25 & 9066164 & 47032.6 & 146.4991 & 0.008831 \\
\hline & 2 & 0.1 & 0.3 & 0.3 & 0.3 & 9264262 & 41338.4 & 141.6119 & 0.008835 \\
\hline & 3 & 0.3 & 0.1 & 0.3 & 0.3 & 8824665 & 30993.68 & 166.3473 & 0.008925 \\
\hline & 4 & 0.3 & 0.3 & 0.1 & 0.3 & 8824665 & 30993.68 & 166.3473 & 0.008925 \\
\hline & 5 & 0.3 & 0.3 & 0.3 & 0.1 & 9066164 & 47032.6 & 146.4991 & 0.008831 \\
\hline
\end{tabular}


Table. 8. Optimum capacity of DGs in the case of $w_{1}=w_{2}=w_{3}=0.33$ and $w_{4}=0$

\begin{tabular}{cccc}
\hline \multicolumn{4}{c}{ DG capacity (kW) } \\
\hline Stage & Bus\#18 & Bus\#32 & Bus\#33 \\
\hline 1 & 210 & 180 & 120 \\
2 & 210 & 180 & 80 \\
3 & 210 & 180 & 30 \\
\hline
\end{tabular}

Table 9: The results of reliability sensitivity analysis

\begin{tabular}{cccccc}
\hline U and U' Factor & No. of Rewiring & Total DG Capacity & Cost (R\$) & ENS (kWh/year) & Power Loss (kW) \\
\hline \hline 0.6 & 10 & 0 & $2.02 E+06$ & 28077.06 & 342.3863296 \\
0.8 & 9 & 0 & 2550602.498 & 36874.5 & 362.3144109 \\
0.9 & 14 & 0 & 2813502.035 & 41612.715 & 316.0013617 \\
1 & 14 & 330 & 2820899.31 & 46063.975 & 373.5441727 \\
1.1 & 8 & 660 & 2955195.355 & 46963.015 & 308.0227299 \\
1.2 & 6 & 990 & 3235392.711 & 49410.78 & 373.6051303 \\
1.4 & 11 & 330 & 3163898.799 & 59739.085 & 322.4029628 \\
\hline
\end{tabular}

Table 10: The result of load sensitivity analysis

\begin{tabular}{cccccc}
\hline Load Factor & No. of Rewiring & Total DG Capacity & Cost (R\$) & ENS (kWh/year) & Power Loss (kW) \\
\hline \hline 0.9 & 8 & 300 & 2793389.333 & 41419 & 274.5548 \\
1 & 14 & 330 & 2820899.31 & 46063.975 & 373.5441727 \\
1.05 & 8 & 690 & 3121500 & 47457 & 503.2966 \\
1.1 & 13 & 570 & 3609300 & 51024 & 496.1497 \\
\hline
\end{tabular}


Table 11.Values of GD, SP and DM for different optimization algorithms in two \& three dimensional Pareto fronts

\begin{tabular}{|c|c|c|c|c|c|c|c|c|c|}
\hline \multirow{2}{*}{$\begin{array}{l}\text { Algorithm } \\
\text { Objective }\end{array}$} & \multicolumn{3}{|c|}{ MPSO } & \multicolumn{3}{|c|}{ PSO } & \multicolumn{3}{|c|}{ GA } \\
\hline & GD & SP & $\mathrm{DM}$ & GD & SP & $\mathrm{DM}$ & GD & $\mathrm{SP}$ & $\mathrm{DM}$ \\
\hline Cost-Loss & 0.0022 & 0.0153 & $3.36 E+14$ & 0.0037 & 0.0218 & $3.33 E+14$ & 0.0082 & 0.0279 & $5.49 \mathrm{E}+13$ \\
\hline Cost-END & 0.0168 & 0.0822 & $1.20 \mathrm{E}+14$ & 0.0109 & 0.0461 & $4.25 \mathrm{E}+13$ & 0.0129 & 0.053 & $3.61 \mathrm{E}+13$ \\
\hline Cost-VSI & 0.0352 & 0.1152 & $1.95 \mathrm{E}+13$ & 0.0381 & 0.0992 & $1.05 \mathrm{E}+13$ & 0.2683 & 0.3678 & $5.10 \mathrm{E}+12$ \\
\hline Cost-END-Loss & 4.75E-04 & 0.0064 & $6.50 \mathrm{E}+14$ & 0.0049 & 0.0241 & $1.97 \mathrm{E}+13$ & 0.0052 & 0.021 & $1.84 \mathrm{E}+13$ \\
\hline Cost-END-VSI & 0.0039 & 0.0168 & $1.06 \mathrm{E}+14$ & 0.0068 & 0.0225 & $4.05 E+13$ & 0.0121 & 0.0225 & $3.17 \mathrm{E}+13$ \\
\hline Cost-Loss-VSI & 0.0027 & 0.0113 & $8.81 \mathrm{E}+13$ & 0.0036 & 0.0242 & $1.03 \mathrm{E}+14$ & 0.0039 & 0.0228 & $8.59 E+13$ \\
\hline END-Loss-VSI & 0.0382 & 0.083 & $7.44 \mathrm{E}+07$ & 0.3057 & 0.5257 & $7.30 \mathrm{E}+07$ & 0.4005 & 0.5787 & $4.13 \mathrm{E}+06$ \\
\hline
\end{tabular}

